for the

communication

entitled

\title{
A Stereoselective Intramolecular Halo-Etherification of Chiral Enamides in the Synthesis of Halogenated Cyclic Ethers.
}

\author{
authored by \\ Changhong Ko, Richard P. Hsung*, Ziyad F. Al-Rashid, John B. Feltenberger, Ting Lu, \\ Jin-Haek Yang, Yonggang Wei, and Craig A. Zificsak \\ Division of Pharmaceutical Sciences \\ Department of Chemistry, University of Wisconsin, Madison, WI 53705-2222
}




\section{Procedure for the Preparation of Chiral Enamides.}

\section{Method A: Dehydrative Condensations.}

To a 100-mL RB-Flask were added $(R)$-4-phenyl-2-oxazolidinone (840.0 mg, 5.15 mmol), the corresponding aldehyde $(1.26 \mathrm{~g}, 5.66 \mathrm{mmol})$ and anhyd toluene $(50 \mathrm{~mL})$. After which, pyridinium $p$-toluenesulfonate $(60.0 \mathrm{mg}, 0.30 \mathrm{mmol})$ was added and the resulting reaction mixture was heated at reflux with azeotropic removal of water for $48 \mathrm{~h}$, and the progress of the reaction was monitored via TLC. Upon disappearance of the starting material, the solution was poured into $50 \mathrm{~mL}$ sat aq $\mathrm{NaHCO}_{3}$ and extracted with $\mathrm{CH}_{2} \mathrm{Cl}_{2}(3 \times 50 \mathrm{~mL})$. The combined organic layers were washed with sat aq $\mathrm{NaCl}(50 \mathrm{~mL})$, dried over $\mathrm{Na}_{2} \mathrm{SO}_{4}$, and concentrated in vасио. Further purification was performed using silica gel flash column chromatography (gradient eluent: 10:1 to $4: 1$ hexane/EtOAc) to afford the pure enamide $6(1.24 \mathrm{~g}, 67 \%)$ as colorless oil.

\section{Characterizations.}

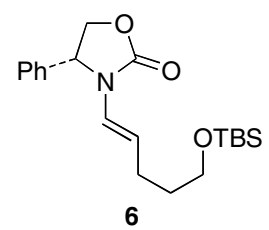

$R_{f}=0.34[20 \% \mathrm{EtOAc} /$ hexane $] ;[\alpha]_{\mathrm{D}}^{23}=-68.7\left(\mathrm{c} 0.49, \mathrm{CHCl}_{3}\right)$;

${ }^{1} \mathrm{H}$ NMR $\left(400 \mathrm{MHz}, \mathrm{CDCl}_{3}\right) \delta-0.01(\mathrm{~s}, 6 \mathrm{H}), 0.87(\mathrm{~s}, 9 \mathrm{H}), 1.42(\mathrm{tt}, J=6.8$ and $7.2 \mathrm{~Hz}, 2 \mathrm{H}), 1.98$ $(\mathrm{q}, J=7.2 \mathrm{~Hz}, 2 \mathrm{H}), 3.43(\mathrm{t}, J=6.8 \mathrm{~Hz}, 2 \mathrm{H}), 4.11(\mathrm{dd}, J=5.2$ and $9.2 \mathrm{~Hz}, 1 \mathrm{H}), 4.62(\mathrm{dt}, J=7.2$ and $14.4 \mathrm{~Hz}, 1 \mathrm{H}), 4.71(\mathrm{dd}, J=9.2$ and $9.2 \mathrm{~Hz}, 1 \mathrm{H}), 5.00(\mathrm{dd}, J=5.2$ and $9.2 \mathrm{~Hz}, 1 \mathrm{H}), 6.60(\mathrm{~d}, J$ $=14.4 \mathrm{~Hz}, 1 \mathrm{H}), 7.23-7.27(\mathrm{~m}, 2 \mathrm{H}), 7.33-7.42(\mathrm{~m}, 3 \mathrm{H}) ;{ }^{13} \mathrm{C} \mathrm{NMR}\left(100 \mathrm{MHz}, \mathrm{CDCl}_{3}\right) \delta-5.4,18.2$, 25.9, 26.2, 32.7, 58.5, 61.9, 70.5, 112.9, 122.8, 125.8, 128.6, 129.2, 138.3, 155.8; IR (neat) cm ${ }^{-1}$ 2928m, 2856m, 1764s, 1672m, 1483m; mass spectrum (APCI): m/e (\% relative intensity) 362 $(\mathrm{M}+\mathrm{H})^{+}(20), 248(100)$; HRMS (MALDI) m/e calcd for $\mathrm{C}_{20} \mathrm{H}_{31} \mathrm{NNaO}_{3} \mathrm{Si}[\mathrm{M}+\mathrm{Na}]^{+} 384.1971$, found 384.2021.

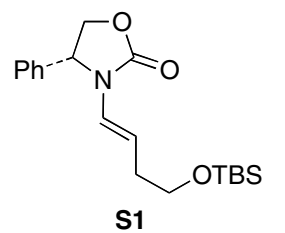

$R_{f}=0.43[33 \%$ EtOAc /hexane $] ; \operatorname{mp~} 53-55^{\circ} \mathrm{C} ;[\alpha]_{\mathrm{D}}^{23}=-68.7\left(\mathrm{c} 3.2, \mathrm{CH}_{2} \mathrm{Cl}_{2}\right)$; 
${ }^{1} \mathrm{H}$ NMR (400 MHz, $\mathrm{CDCl}_{3}$ ) $\delta 0.00$ (s, 3H), 0.02 (s, 3H), 0.89 (s, 9H), 2.16 (ddd, $J=6.4,6.4$, and $9.2 \mathrm{~Hz}, 2 \mathrm{H}), 3.51(\mathrm{dt}, J=3.6$ and $6.4 \mathrm{~Hz}, 2 \mathrm{H}), 4.16(\mathrm{dd}, J=5.2$ and $8.4 \mathrm{~Hz}, 1 \mathrm{H}), 4.71(\mathrm{dt}, J$ $=5.2$ and $14.8 \mathrm{~Hz}, 1 \mathrm{H}), 4.75(\mathrm{dd}, J=8.8$ and $8.8 \mathrm{~Hz}, 1 \mathrm{H}), 5.04(\mathrm{dd}, J=5.2$ and $8.8 \mathrm{~Hz}, 1 \mathrm{H}), 6.69$ $(\mathrm{d}, J=14.8 \mathrm{~Hz}, 1 \mathrm{H}), 7.29-7.32(\mathrm{~m}, 2 \mathrm{H}), 7.38-7.47(\mathrm{~m}, 3 \mathrm{H}) ;{ }^{13} \mathrm{C} \mathrm{NMR}\left(100 \mathrm{MHz}, \mathrm{CDCl}_{3}\right) \delta-5.1$, $18.5,26.1,33.8,58.9,63.3,70.8,110.0,124.3,126.1,129.0,129.6,138.5,156.0$; IR (thin film) $\mathrm{cm}^{-1} 3050 \mathrm{w}, 2915 \mathrm{~m}, 1757 \mathrm{~s}, 1405 \mathrm{~s}, 1225 \mathrm{~s}$; mass spectrum (APCI): $\mathrm{m} / \mathrm{e}$ (\% relative intensity) 348 $(\mathrm{M}+\mathrm{H})^{+}(100)$.

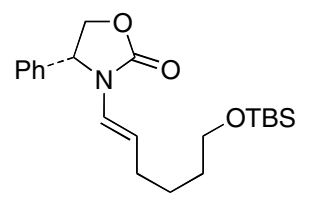

S2

$R_{f}=0.40[20 \%$ EtOAc /hexane $] ;[\alpha]_{\mathrm{D}}^{23}=-68.1\left(c 0.93, \mathrm{CCl}_{4}\right)$;

${ }^{1} \mathrm{H}$ NMR $\left(500 \mathrm{MHz}, \mathrm{CDCl}_{3}\right) \delta 0.00(\mathrm{~s}, 3 \mathrm{H}), 0.08(\mathrm{~s}, 3 \mathrm{H}), 0.86(\mathrm{~s}, 9 \mathrm{H}), 1.21-1.24(\mathrm{~m}, 2 \mathrm{H}), 1.30-$ $1.32(\mathrm{~m}, 2 \mathrm{H}), 1.28-1.90(\mathrm{~m}, 2 \mathrm{H}), 3.49(\mathrm{t}, J=6.5 \mathrm{~Hz}, 2 \mathrm{H}), 4.10(\mathrm{dd}, J=5.0$ and $9.0 \mathrm{~Hz}, 1 \mathrm{H}), 4.59$ (ddd, $J=7.0,7.0$, and $15.0 \mathrm{~Hz}, 1 \mathrm{H}), 4.68(\mathrm{dd}, J=9.0$ and $9.0 \mathrm{~Hz}, 1 \mathrm{H}), 4.98(\mathrm{dd}, J=5.0$ and 9.0 $\mathrm{Hz}, 1 \mathrm{H}), 6.56(\mathrm{~d}, J=15.0 \mathrm{~Hz}, 1 \mathrm{H}) 7.22-7.39(\mathrm{~m}, 5 \mathrm{H}) ;{ }^{13} \mathrm{C} \mathrm{NMR}\left(125 \mathrm{MHz}, \mathrm{CDCl}_{3}\right) \delta 0.0,0.0$, 1.7, 23.6, 31.26, 31.3, 31.3, 31.3, 35.0, 37.2, 63.9, 68.2, 75.8, 118.9, 128.0, 131.2, 131.2, 134.0, 133.6, 134.6, 143.6, 161.1; IR (neat) $\mathrm{cm}^{-1} 2930 \mathrm{~s}, 2857 \mathrm{~s}, 1760 \mathrm{~s}, 1671 \mathrm{w}, 1405 \mathrm{~s}, 1089 \mathrm{~s}, 834 \mathrm{~s}$; mass spectrum (APCI): $\mathrm{m} / \mathrm{e}$ (\% relative intensity) $376(\mathrm{M}+\mathrm{H})^{+}(100), 262(45)$.

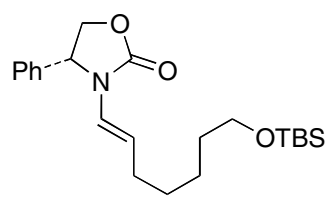

S3

$R_{f}=0.59\left[30 \%\right.$ EtOAc /hexane]; $[\alpha]_{\mathrm{D}}^{23}=-55.1\left(\right.$ c 3.67, $\left.\mathrm{CHCl}_{3}\right)$;

${ }^{1} \mathrm{H}$ NMR $\left(400 \mathrm{MHz}, \mathrm{CDCl}_{3}\right) \delta 0.04(\mathrm{~s}, 6 \mathrm{H}), 0.89(\mathrm{~s}, 9 \mathrm{H}), 1.09-1.25(\mathrm{~m}, 4 \mathrm{H}), 1.41$ (dddd, $J=6.8$ $\mathrm{Hz}, 2 \mathrm{H}), 1.84-1.93(\mathrm{~m}, 2 \mathrm{H}), 3.53(\mathrm{t}, J=6.8 \mathrm{~Hz}, 2 \mathrm{H}), 4.11(\mathrm{dd}, J=5.6$ and $8.8 \mathrm{~Hz}, 1 \mathrm{H}), 4.61(\mathrm{dt}$, $J=7.2$ and $14.8 \mathrm{~Hz}, 1 \mathrm{H}), 4.70(\mathrm{t}, J=9.2 \mathrm{~Hz}, 1 \mathrm{H}), 5.01(\mathrm{dd}, J=5.2$ and $9.2 \mathrm{~Hz}, 1 \mathrm{H}), 6.55(\mathrm{~d}, J=$ $14.8 \mathrm{~Hz}, 1 \mathrm{H}), 7.24-7.42(\mathrm{~m}, 5 \mathrm{H}) ;{ }^{13} \mathrm{C}$ NMR $\left(100 \mathrm{MHz}, \mathrm{CDCl}_{3}\right) \delta-5.0,18.6,25.2,26.2,29.8$, $30.1,32.8,58.9,63.4,70.7,113.9,122.8,126.1,128.9,129.5,138.6,156.0$; IR (neat) $\mathrm{cm}^{-1} 2937 \mathrm{w}$, $1755 \mathrm{~m}, 1407 \mathrm{~m}, 1264 \mathrm{~m}$; mass spectrum (APCI): $\mathrm{m} / \mathrm{e}$ (\% relative intensity) $390(\mathrm{M}+\mathrm{H})^{+}(100)$, 276 (7). 


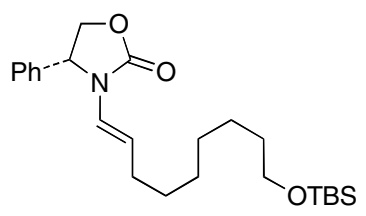

S4

$R_{f}=0.50\left[25 \%\right.$ EtOAc /hexane]; $[\alpha]_{\mathrm{D}}^{23}=-59.3\left(c 0.27, \mathrm{CCl}_{4}\right)$;

${ }^{1} \mathrm{H}$ NMR $\left(500 \mathrm{MHz}, \mathrm{CDCl}_{3}\right) \delta 0.00(\mathrm{~s}, 6 \mathrm{H}), 0.85(\mathrm{~s}, 9 \mathrm{H}), 1.03-1.05(\mathrm{~m}, 2 \mathrm{H}), 1.09-1.21(\mathrm{~m}, 6 \mathrm{H})$, $1.39-1.43(\mathrm{~m}, 2 \mathrm{H}), 1.82-1.85(\mathrm{~m}, 2 \mathrm{H}), 3.53(\mathrm{t}, J=3.2 \mathrm{~Hz}, 2 \mathrm{H}), 4.05(\mathrm{dd}, J=5.6$ and $8.8 \mathrm{~Hz}, 1 \mathrm{H})$, $4.55(\mathrm{ddd}, J=7.2,7.2$, and $14.4 \mathrm{~Hz}, 1 \mathrm{H}), 4,65(\mathrm{dd}, J=8.8$ and $8.8 \mathrm{~Hz}, 1 \mathrm{H}), 4.95(\mathrm{dd}, J=5.6$ and $9.2 \mathrm{~Hz}, 1 \mathrm{H}), 6.50(\mathrm{~d}, J=14.4 \mathrm{~Hz}, 1 \mathrm{H}), 7.19-7.23(\mathrm{~m}, 2 \mathrm{H}), 7.29-7.36(\mathrm{~m}, 3 \mathrm{H}) ;{ }^{13} \mathrm{C}$ NMR $(125$ $\left.\mathrm{MHz}, \mathrm{CDCl}_{3}\right) \delta$ 0.0, 0.0, 23.6, 30.9, 31.2, 31.2, 31.24 33.9, 34.4, 34.9, 35.1, 38.1, 63.9, 68.5, 75.7, 119.1, 127.7, 131.1, 131.1, 133.9, 134.5, 134.5, 143.6, 161.1; IR (neat) $\mathrm{cm}^{-1} 2930 \mathrm{~s}, 2857 \mathrm{~s}$, 1760s, 1671w, 1407s, 1093s, 836s; mass spectrum (APCI): m/e (\% relative intensity) 418 (M + $\mathrm{H})^{+}(100), 304(10)$.

\section{Method B: Lindlar Hydrogenations of Ynamides.}

(a) Preparation of Ynamides. The respective 1-bromoalkyne (1.0 equiv) in toluene $(0.4 \mathrm{M})$ was combined with the corresponding chiral oxazolidinone (1.05 equiv), $\mathrm{CuSO}_{4} .5 \mathrm{H}_{2} \mathrm{O}$ (0.15 equiv), 1,10-phenanthroline ( 0.30 equiv), and $\mathrm{K}_{2} \mathrm{CO}_{3}$ (2.0 equiv). The reaction mixture stirred at $\mathrm{rt}$ for $5 \mathrm{~min}$ and then heated in an oil bath at $80-85^{\circ} \mathrm{C}$ for $24 \mathrm{~h}$ while being monitored via TLC analysis. Upon completion, the reaction mixture was cooled to $\mathrm{rt}$ and diluted with EtOAc and filtered through Celite ${ }^{\mathrm{TM}}$, and the filtrate was concentrated in vacuo. The crude products were purified by silica gel flash column chromatography [gradient elution: EtOAc in hexanes] to afford the desired ynamide.

(b) Hydrogenate of Ynamides. To a stirred solution of the ynamide prepared above in $\mathrm{CH}_{2} \mathrm{Cl}_{2}(0.01 \mathrm{M})$ at $\mathrm{rt}$, was added Lindlar's catalyst $\left(\mathrm{Pd} / \mathrm{CaCO}_{3} / \mathrm{Pb}\right)(5 \mathrm{~mol} \%)$. The heterogeneous mixture was stirred under 1 atm of $\mathrm{H}_{2}$, and the progress of the reaction was monitored via TLC. Upon completion, the reaction mixture was diluted with EtOAc and filtered. The filtrate was concentrated in vacuo leading to quantitative isolation of the desired hydrogenated product. 


\section{Characterizations.}

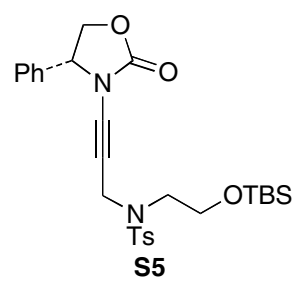

$R_{f}=0.54\left[50 \%\right.$ EtOAc/hexanes]; $[\alpha]_{\mathrm{D}}{ }^{25}=-89.9\left(c 1.07, \mathrm{CH}_{2} \mathrm{Cl}_{2}\right)$;

${ }^{1} \mathrm{H}$ NMR $\left(500 \mathrm{MHz}, \mathrm{CDCl}_{3}\right) \delta-0.00$ (s, 6H), 0.84 (s, 9H), 2.45 (s, 3H), 2.73 (dddd, $J=14.5,6.0$, $5.0,0.5 \mathrm{~Hz}, 1 \mathrm{H}), 3.01$ (dddd, $J=14.5,5.5,5.5,1.0 \mathrm{~Hz}, 1 \mathrm{H}), 3.61$ (ddd, $J=10.5,5.5,5.5 \mathrm{~Hz}, 1 \mathrm{H}$ ), $3.65(\mathrm{ddd}, J=10.5,6.5,5.5 \mathrm{~Hz}, 1 \mathrm{H}), 4.17(\mathrm{dd}, J=9.0,7.0 \mathrm{~Hz}, 1 \mathrm{H}), 4.23(\mathrm{dd}, J=18.5,0.5 \mathrm{~Hz}$, $1 \mathrm{H}), 4.35(\mathrm{dd}, J=18.5,1.0 \mathrm{~Hz}, 1 \mathrm{H}), 4.66(\mathrm{dd}, J=9.0,9.0 \mathrm{~Hz}, 1 \mathrm{H}), 4.81$ (dd, $J=8.5,7.0 \mathrm{~Hz}, 1 \mathrm{H})$, $7.10-7.12(\mathrm{~m}, 2 \mathrm{H}), 7.28(\mathrm{dq}, J=8.5,0.5 \mathrm{~Hz}, 2 \mathrm{H}), 7.36-7.39(\mathrm{~m}, 3 \mathrm{H})$, and $7.69(\mathrm{~d}, J=8.5 \mathrm{~Hz}$, $2 \mathrm{H}) ;{ }^{13} \mathrm{C} \mathrm{NMR}\left(100 \mathrm{MHz}, \mathrm{CDCl}_{3}\right) \delta-5.3,18.3,21.7,26.0,38.8,47.9,61.7,62.7,67.0,70.8,74.4$, 126.7, 127.9, 129.4, 129.6, 129.7, 136.0, 136.4, 143.4, 155.4; IR (neat) $\mathrm{cm}^{-1} 2955 \mathrm{w}, 1782 \mathrm{~s}$, $1472 \mathrm{w}, 1407 \mathrm{~m}, 1345 \mathrm{~m}, 1162 \mathrm{~s}, 1105 \mathrm{~m}$, and 838m; mass spectrum (APCI): m/e (\% relative intensity) 529 (100) $(\mathrm{M}+\mathrm{H})^{+}$, and 415 (25); HRMS (MALDI) m/e calcd for $\mathrm{C}_{27} \mathrm{H}_{36} \mathrm{~N}_{2} \mathrm{O}_{5} \mathrm{SSiNa}^{+}$ $\left(\mathrm{M}+\mathrm{Na}^{+}\right), 551.2006$ found 551.1919 .

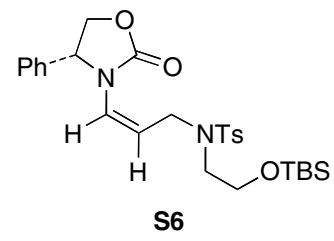

$R_{f}=0.50[50 \%$ EtOAc /hexane $] ;[\alpha]_{\mathrm{D}}^{23}=-64.1\left(c 0.92, \mathrm{CH}_{2} \mathrm{Cl}_{2}\right)$;

${ }^{1} \mathrm{H}$ NMR (400 MHz, $\mathrm{CDCl}_{3}$ ) $\delta 0.02$ (s, 6H), 0.86 (s, 9H), 2.41 (s, 3H), 3.04 (ddd, $J=6.8,6.8$, and $14.4 \mathrm{~Hz}, 1 \mathrm{H}), 3.12(\mathrm{ddd}, J=6.0,6.0$, and $14.0 \mathrm{~Hz}, 1 \mathrm{H}), 3.63-3.69(\mathrm{~m}, 2 \mathrm{H}), 3.76(\mathrm{ddd}, J=$ 1.6, 5.6, and $16.4 \mathrm{~Hz}, 1 \mathrm{H}), 4.01$ (ddd, $J=1.6,8.0$, and $16.4 \mathrm{~Hz}, 1 \mathrm{H}), 4.15(\mathrm{dd}, J=6.0$ and $8.8 \mathrm{~Hz}$, $1 \mathrm{H}), 4.69$ (dd, $J=8.8$ and $8.8 \mathrm{~Hz}, 1 \mathrm{H}), 4.93(\mathrm{ddd}, J=5.6,8.0$, and $9.2 \mathrm{~Hz}, 1 \mathrm{H}), 5.12(\mathrm{dd}, J=6.0$ and $8.8 \mathrm{~Hz}, 1 \mathrm{H}), 5.87$ (ddd, $J=1.6,1.6$, and $9.2 \mathrm{~Hz}, 1 \mathrm{H}), 7.24-7.28(\mathrm{~m}, 4 \mathrm{H}), 7.37-7.41(\mathrm{~m}$, $3 \mathrm{H})$, and $7.60(\mathrm{~d}, J=8.0 \mathrm{~Hz}, 2 \mathrm{H}) ;{ }^{13} \mathrm{C} \mathrm{NMR}\left(100 \mathrm{MHz}, \mathrm{CDCl}_{3}\right) \delta-5.2,18.4,21.7,26.1,46.8$, 49.8, 61.2, 62.6, 70.4, 116.8, 123.6, 126.6, 127.4, 129.4, 129.6, 129.8, 137.0, 137.9, 143.5, 156.3; IR (neat) $\mathrm{cm}^{-1} 3035 \mathrm{w}, 2955 \mathrm{w}, 1765 \mathrm{~s}, 1396 \mathrm{~m}, 1342 \mathrm{~m}, 1159 \mathrm{~s}, 1091 \mathrm{~m}$, and $837 \mathrm{~m}$; mass spectrum (APCI): $\mathrm{m} / \mathrm{e}\left(\%\right.$ relative intensity) $531(\mathrm{M}+\mathrm{H})^{+}(10), 375(50), 202$ (100). 


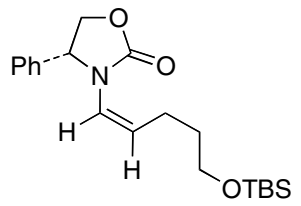

6-Z

$R_{f}=0.57[50 \% \mathrm{EtOAc} /$ hexanes $] ;[\alpha]_{\mathrm{D}}^{25}=-57.9\left(c 0.94, \mathrm{CH}_{2} \mathrm{Cl}_{2}\right)$;

${ }^{1} \mathrm{H}$ NMR (400 MHz, $\left.\mathrm{CDCl}_{3}\right) \delta 0.04(\mathrm{~s}, 6 \mathrm{H}), 0.89$ (s, 9H), 1.45 (m, 2H), 1.99 (ddddd, $J=15.2$, 8.4, 8.4, 6.8, $1.6 \mathrm{~Hz}, 1 \mathrm{H}), 2.15$ (ddddd, $J=15.2,8.4,8.4,7.2,1.6 \mathrm{~Hz}, 1 \mathrm{H}), 3.47-3.57$ (m, 2H), $4.15(\mathrm{dd}, J=8.8,5.2 \mathrm{~Hz}, 1 \mathrm{H}), 4.67(\mathrm{dd}, J=8.8,8.8 \mathrm{~Hz}, 1 \mathrm{H}), 5.00$ (ddd, $J=8.8,8.0,7.2 \mathrm{~Hz}, 1 \mathrm{H})$, $5.14(\mathrm{dd}, J=8.8,5.2 \mathrm{~Hz}, 1 \mathrm{H}), 5.93(\mathrm{dt}, J=8.8,1.2 \mathrm{~Hz}, 1 \mathrm{H}), 7.23-7.26(\mathrm{~m}, 2 \mathrm{H}), 7.33-7.42(\mathrm{~m}$, $3 \mathrm{H}) ;{ }^{13} \mathrm{C} \mathrm{NMR}\left(100 \mathrm{MHz}, \mathrm{CDCl}_{3}\right) \delta-5.1,18.5,24.0,26.2,31.6,32.5,45.3,61.1,62.6,70.3$, 121.4, 122.5, 126.4, 129.1, 129.4, 138.7, 156.7; IR (neat) $\mathrm{cm}^{-1} 3034 \mathrm{w}, 2953 \mathrm{w}, 1757 \mathrm{~s}, 1393 \mathrm{~m}$, and 834s; mass spectrum (APCI): m/e (\% relative intensity) $362(40)(\mathrm{M}+\mathrm{H})^{+}$, and $248(100)$; HRMS (MALDI) m/e calcd for $\left(\mathrm{C}_{20} \mathrm{H}_{32} \mathrm{NO}_{3} \mathrm{Si}\right)^{+}\left(\mathrm{M}+\mathrm{H}^{+}\right)$362.2146, found 362.2171.

\section{Method C. Amidative Cross-Coupling.}

To a 5-mL RB-Flask were added $(R)$-4-diphenylmethyl-2-oxazolidinone 16 (150.0 mg, $0.59 \mathrm{mmol})$, vinyl halide $15(152.0 \mathrm{mg}, 0.49 \mathrm{mmol})$, CuI (10.0 mg, $0.050 \mathrm{mmol}), N, N-$ dimethylglycine $(14.0 \mathrm{mg}, 0.10 \mathrm{mmol})$, and $\mathrm{Cs}_{2} \mathrm{CO}_{3}(322.0 \mathrm{mg}, 0.99 \mathrm{mmol})$. The flask was evacuated and backfilled with nitrogen at $0{ }^{\circ} \mathrm{C}$ and dioxane $(2 \mathrm{~mL})$ was added. The resulting reaction mixture was warmed up to $80{ }^{\circ} \mathrm{C}$ and stirred at that temperature for $24 \mathrm{~h}$ while being monitored via TLC. Upon disappearance of the starting material, the reaction mixture was cooled to room temperature and diluted with EtOAc $(10 \mathrm{~mL})$. The solution was filtrated through a silica

gel eluting with ethyl acetate. The filtrate was concentrated in vacuo. Further purification was performed using silica gel flash column chromatography (gradient eluent: 10:1 to 4:1 hexane/EtOAc) to afford the pure corresponding enamide 17 (153.0 mg, 70\%) as colorless oil.

\section{CharacteriZations.}

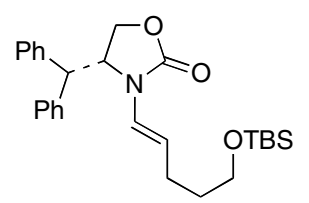

17

$R_{\mathrm{f}}=0.39[20 \% \mathrm{EtOAc} /$ hexane $\left.] ; \alpha\right]_{\mathrm{D}}^{23}=-123.0\left(\mathrm{c} 0.49, \mathrm{CHCl}_{3}\right)$;

${ }^{1} \mathrm{H}$ NMR (400 MHz, $\left.\mathrm{CDCl}_{3}\right) \delta 0.45(\mathrm{~s}, 6 \mathrm{H}), 0.89(\mathrm{~s}, 9 \mathrm{H}), 1.57(\mathrm{tt}, J=6.4$ and $7.2 \mathrm{~Hz}, 2 \mathrm{H}), 2.11$ 
$(\mathrm{q}, J=7.2 \mathrm{~Hz}, 2 \mathrm{H}), 3.61(\mathrm{t}, J=6.4 \mathrm{~Hz}, 2 \mathrm{H}), 4.35(\mathrm{dd}, J=3.2$ and $8.8 \mathrm{~Hz}, 1 \mathrm{H}), 4.46(\mathrm{dd}, J=8.4$ and $8.8 \mathrm{~Hz}, 1 \mathrm{H}), 4.73(\mathrm{~d}, J=3.6 \mathrm{~Hz}, 1 \mathrm{H}), 4.79(\mathrm{ddd}, J=3.2,3.6$, and $8.4 \mathrm{~Hz}, 1 \mathrm{H}), 4.98(\mathrm{dt}, J=$ 7.2 and $14.8 \mathrm{~Hz}, 1 \mathrm{H}), 6.50(\mathrm{~d}, J=14.8 \mathrm{~Hz}, 1 \mathrm{H}), 7.02-7.05(\mathrm{~m}, 2 \mathrm{H}), 7.09-7.12(\mathrm{~m}, 2 \mathrm{H}), 7.23-7.32$ $(\mathrm{m}, 6 \mathrm{H}) ;{ }^{13} \mathrm{C}$ NMR $\left(100 \mathrm{MHz}, \mathrm{CDCl}_{3}\right) \delta-5.1,18.5,26.1,26.8,33.3,48.7,56.9,62.6,64.9,112.4$, 123.2, 127.4, 127.8, 128.7, 128.9, 129.1, 129.3, 138.5, 140.1, 155.4; IR (neat) $\mathrm{cm}^{-1} 3010 \mathrm{~m}$, $2934 \mathrm{~m}, 1785 \mathrm{~s}, 1417 \mathrm{~s}, 1265 \mathrm{~s}$; mass spectrum (APCI): $\mathrm{m} / \mathrm{e}\left(\%\right.$ relative intensity) $452.2(\mathrm{M}+\mathrm{H})^{+}$ (100).

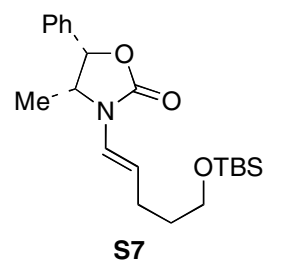

$R_{f}=0.50[20 \% \mathrm{EtOAc} /$ hexane $] ;[\alpha]_{\mathrm{D}}^{23}=+21.6\left(\mathrm{c} 0.63, \mathrm{CHCl}_{3}\right)$;

${ }^{1} \mathrm{H}$ NMR $\left(400 \mathrm{MHz}, \mathrm{CDCl}_{3}\right) \delta 0.05(\mathrm{~s}, 6 \mathrm{H}), 0.83(\mathrm{~d}, J=6.8 \mathrm{~Hz}, 3 \mathrm{H}), 0.90(\mathrm{~s}, 9 \mathrm{H}), 1.62(\mathrm{tt}, J=$ 6.4 and $6.8 \mathrm{~Hz}, 2 \mathrm{H}), 2.15(\mathrm{dt}, J=6.8$ and $7.2 \mathrm{~Hz}, 2 \mathrm{H}), 3.63(\mathrm{t}, J=6.4 \mathrm{~Hz}, 2 \mathrm{H}), 4.33(\mathrm{dq}, J=6.8$ and $7.6 \mathrm{~Hz}, 1 \mathrm{H}), 4.96(\mathrm{dt}, J=7.2$ and $14.8 \mathrm{~Hz}, 1 \mathrm{H}), 5.67(\mathrm{~d}, J=7.6 \mathrm{~Hz}, 1 \mathrm{H}), 6.58(\mathrm{~d}, J=14.8$ $\mathrm{Hz}, 1 \mathrm{H}), 7.31-7.43(\mathrm{~m}, 5 \mathrm{H}) ;{ }^{13} \mathrm{C} \mathrm{NMR}\left(100 \mathrm{MHz}, \mathrm{CDCl}_{3}\right) \delta-5.1,13.2,18.5,26.1,26.7,33.3$, 54.5, 62.6, 79.1, 111.9, 122.9, 126.1, 128.7, 128.9, 134.5, 154.6; IR (thin film) $\mathrm{cm}^{-1} 3058 \mathrm{~m}$, 2990m, 1752s, 1400s, 1253s; mass spectrum (APCI): m/e (\% relative intensity) $376(\mathrm{M}+\mathrm{H})^{+}$ (40), 276 (50), 262 (100).

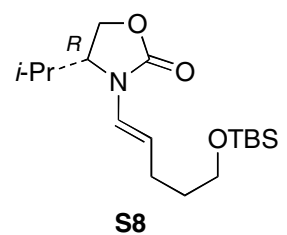

$R_{f}=0.35[20 \% \mathrm{EtOAc} /$ hexane $] ;[\alpha]_{\mathrm{D}}^{23}=-13.1\left(\mathrm{c} 0.53, \mathrm{CHCl}_{3}\right) ;$

${ }^{1} \mathrm{H}$ NMR (400 MHz, $\left.\mathrm{CDCl}_{3}\right) \delta 0.03(\mathrm{~s}, 6 \mathrm{H}), 0.38(\mathrm{~d}, J=6.8 \mathrm{~Hz}, 3 \mathrm{H}), 0.88(\mathrm{~s}, 9 \mathrm{H}), 0.90(\mathrm{~d}, J=$ $6.8 \mathrm{~Hz}, 3 \mathrm{H}), 1.59(\mathrm{tt}, J=6.4$ and $7.2 \mathrm{~Hz}, 2 \mathrm{H}), 2.11(\mathrm{q}, J=7.2 \mathrm{~Hz}, 2 \mathrm{H}), 3.38(\mathrm{dh}, J=3.2$ and 6.8 $\mathrm{Hz}, 1 \mathrm{H}), 3.60$ (t, $J=6.4 \mathrm{~Hz}, 2 \mathrm{H}), 3.99$ (ddd, $J=3.2,3.6$, and $8.8 \mathrm{~Hz}, 1 \mathrm{H}), 4.19$ (dd, $J=3.6$ and $8.8 \mathrm{~Hz}, 1 \mathrm{H}), 4.24(\mathrm{dd}, J=8.8$ and $8.8 \mathrm{~Hz}, 1 \mathrm{H}), 4.96(\mathrm{dt}, J=7.2$ and $14.8 \mathrm{~Hz}, 1 \mathrm{H}), 6.50(\mathrm{~d}, J=$ $14.8 \mathrm{~Hz}, 1 \mathrm{H}) ;{ }^{13} \mathrm{C}$ NMR $\left(100 \mathrm{MHz}, \mathrm{CDCl}_{3}\right) \delta-5.2,14.0,18.0,18.4,26.0,26.1,26.2,26.7,33.2$, 58.6, 62.5, 63.0, 111.8, 123.0; IR (neat) $\mathrm{cm}^{-1} 3058 \mathrm{~m}, 2955 \mathrm{~m}, 1754 \mathrm{~s}, 1412 \mathrm{~s}, 1097 \mathrm{~s}$; mass spectrum (APCI): m/e (\% relative intensity) $328(\mathrm{M}+\mathrm{H})^{+}(40), 228(30), 214.2(100), 130$ (20). 


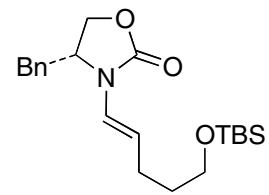

s9

$R_{f}=0.32[20 \%$ EtOAc /hexane $] ;[\alpha]_{\mathrm{D}}^{23}=-26.6\left(\mathrm{c} 0.61, \mathrm{CHCl}_{3}\right) ;$

${ }^{1} \mathrm{H}$ NMR (400 MHz, $\left.\mathrm{CDCl}_{3}\right) \delta 0.00(\mathrm{~s}, 6 \mathrm{H}), 0.84(\mathrm{~s}, 9 \mathrm{H}), 1.60(\mathrm{tt}, J=6.8$ and $7.2 \mathrm{~Hz}, 2 \mathrm{H}), 2.13$ (dddt, $J=1.2,2.0,7.2$, and $8.0 \mathrm{~Hz}, 2 \mathrm{H}), 2.71(\mathrm{dd}, J=8.8$ and $14.4 \mathrm{~Hz}, 1 \mathrm{H}), 3.15(\mathrm{dd}, J=3.2$ and $14.4 \mathrm{~Hz}, 1 \mathrm{H}), 3.59(\mathrm{t}, J=6.8 \mathrm{~Hz}, 2 \mathrm{H}), 4.10(\mathrm{dd}, J=2.8$ and $8.0 \mathrm{~Hz}, 1 \mathrm{H}), 4.15(\mathrm{dd}, J=8.0$ and 8.0 $\mathrm{Hz}, 1 \mathrm{H}), 4.19$ (dddd, $J=2.8,3.2,8.0$, and $8.8 \mathrm{~Hz}, 1 \mathrm{H}), 5.04(\mathrm{dt}, J=7.2$ and $14.8 \mathrm{~Hz}, 1 \mathrm{H}), 6.53$ $(\mathrm{dt}, J=1.2$ and $14.8 \mathrm{~Hz}, 1 \mathrm{H}), 7.09-7.1(\mathrm{~m}, 2 \mathrm{H}), 7.12-7.24(\mathrm{~m}, 1 \mathrm{H}), 7.25-7.30(\mathrm{~m}, 2 \mathrm{H}) ;{ }^{13} \mathrm{C} \mathrm{NMR}$ $\left(100 \mathrm{MHz}, \mathrm{CDCl}_{3}\right) \delta$ 0.0, 23.6, 31.2, 31.8, 38.4, 41.4, 60.3, 67.6, 71.7, 116.9, 128.2, 132.6, 134.2, 134.5, 140.6, 160.4; IR (neat) $\mathrm{cm}^{-1} 3056 \mathrm{~m}, 2986 \mathrm{~m}, 1755 \mathrm{~s}, 1419 \mathrm{~s}, 1265 \mathrm{~s}$; mass spectrum (APCI): $\mathrm{m} / \mathrm{e}\left(\%\right.$ relative intensity) $376(\mathrm{M}+\mathrm{H})^{+}(100), 376(30)$.

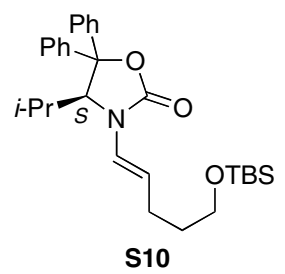

$R_{f}=0.41[20 \%$ EtOAc/hexanes $] ; \operatorname{mp~} 108-109^{\circ} \mathrm{C} ;[\alpha]_{\mathrm{D}}{ }^{23}=-217.0\left(c 0.26, \mathrm{CH}_{2} \mathrm{Cl}_{2}\right)$;

${ }^{1} \mathrm{H}$ NMR $\left(400 \mathrm{MHz}, \mathrm{CDCl}_{3}\right) \delta 0.03(\mathrm{~s}, 6 \mathrm{H}), 0.74(\mathrm{~d}, 3 \mathrm{H}, J=6.8 \mathrm{~Hz}), 0.89(\mathrm{~s}, 9 \mathrm{H}), 1.00(\mathrm{~d}, J=$ $7.2 \mathrm{~Hz}, 3 \mathrm{H}$ ), 1.60 (dddd, $J=6.4,6.4,6.4$, and $6.4 \mathrm{~Hz}, 2 \mathrm{H}$ ), 1.97 (d sept, $J=2.4$ and $7.2 \mathrm{~Hz}, 1 \mathrm{H}$ ), 2.10 (dddd, $J=1.2,7.2,7.2$, and 7.2,Hz, $2 \mathrm{H}), 3.60(\mathrm{dd}, J=6.4$ and $6.4 \mathrm{~Hz}, 2 \mathrm{H}), 4.68(\mathrm{~d}, J=2.4$ $\mathrm{Hz}, 1 \mathrm{H}), 5.25$ (ddd, $J=7.2,7.2$, and $14.6 \mathrm{~Hz}, 1 \mathrm{H}), 6.48$ (ddd, $J=1.2,1.2$, and $14.6 \mathrm{~Hz}, 1 \mathrm{H}), 7.25$ $-7.28(\mathrm{~m}, 2 \mathrm{H}), 7.33(\mathrm{dd}, J=7.6$ and $7.6 \mathrm{~Hz}, 4 \mathrm{H}), 7.42(\mathrm{~d}, J=7.6 \mathrm{~Hz}, 2 \mathrm{H})$, and $7.47(\mathrm{~d}, J=7.6$ $\mathrm{Hz}, 2 \mathrm{H}) ;{ }^{13} \mathrm{C}$ NMR $\left(100 \mathrm{MHz}, \mathrm{CDCl}_{3}\right) \delta-5.1,16.4,18.5,23.1,26.2,26.6,30.5,33.1,62.5,66.5$, $88.9,113.3,124.7,125.7,126.4,127.9,128.4,128.5,128.9,138.9$, 143.8, and 154.6; IR (thin film) $\mathrm{cm}^{-1} 3064 \mathrm{w}, 2957 \mathrm{w}, 1741 \mathrm{~s}, 1674 \mathrm{w}, 1415 \mathrm{~m}, 1254 \mathrm{w}, 1102 \mathrm{~m}$, and $839 \mathrm{~m}$; mass spectrum (APCI): $\mathrm{m} / \mathrm{e}\left(\%\right.$ relative intensity) $480(30)(\mathrm{M}+\mathrm{H})^{+}, 366$ (100); HRMS (MALDI-MS) m/e calcd for $\mathrm{C}_{29} \mathrm{H}_{41} \mathrm{NO}_{3} \mathrm{SiNa}^{+}[\mathrm{M}+\mathrm{Na}]^{+}$502.2748, found 502.2850. 


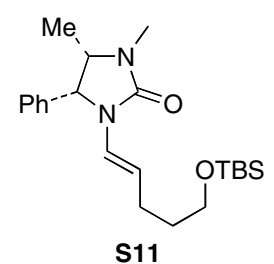

$R_{f}=0.43[33 \% \mathrm{EtOAc} /$ hexane $] ; \operatorname{mp} 60-61{ }^{\circ} \mathrm{C} ;[\alpha]_{\mathrm{D}}{ }^{23}=-31.2\left(\mathrm{c} 0.65, \mathrm{CHCl}_{3}\right)$;

${ }^{1} \mathrm{H}$ NMR $\left(400 \mathrm{MHz}, \mathrm{CDCl}_{3}\right) \delta-0.05$ (s, 3H), -0.04 (s, 3H), 0.73 (d, J=6.8 Hz, 3H), 0.83 (s, 9H), 1.35 (tt, $J=6.8$ and $7.2 \mathrm{~Hz}, 2 \mathrm{H}), 1.89(\mathrm{dt}, J=7.2$ and $7.6 \mathrm{~Hz}, 2 \mathrm{H}), 2.76(\mathrm{~s}, 3 \mathrm{H}), 3.33-3.42(\mathrm{~m}$, $2 \mathrm{H}), 3.81(\mathrm{dt}, J=6.8$ and $8.8 \mathrm{~Hz}, 1 \mathrm{H}), 4.27(\mathrm{dt}, J=7.2$ and $14.8 \mathrm{~Hz}, 1 \mathrm{H}), 4.77(\mathrm{~d}, J=8.8 \mathrm{~Hz}$, $1 \mathrm{H}), 6.72(\mathrm{~d}, J=14.8 \mathrm{~Hz}, 1 \mathrm{H}), 7.05-7.10(\mathrm{~m}, 2 \mathrm{H}), 7.23-7.32(\mathrm{~m}, 3 \mathrm{H}) ;{ }^{13} \mathrm{C} \mathrm{NMR}(100 \mathrm{MHz}$, $\left.\mathrm{CDCl}_{3}\right) \delta-5.1,15.4,18.5,26.1,26.5,29.0,33.5,55.9,60.8,62.4,108.4,123.8,128.2,128.6$, 135.9, 158.2; IR (thin film) $\mathrm{cm}^{-1} 2954 \mathrm{w}, 2859 \mathrm{w}, 1707 \mathrm{~s}, 1436 \mathrm{~s}, 1270 \mathrm{~s}, 1072 \mathrm{~m}$; mass spectrum (LC-MS): m/e (\% relative intensity) $389(\mathrm{M}+\mathrm{H})^{+}(100), 300$ (60), 216 (30).

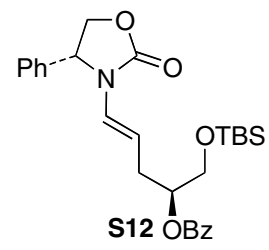

$\mathrm{R}_{\mathrm{f}}=0.41[20 \% \mathrm{EtOAc} /$ hexane $] ; \operatorname{mp} 96-98^{\circ} \mathrm{C} ;[\alpha]_{\mathrm{D}}^{23}=-77.7\left(\mathrm{c} 0.66, \mathrm{CHCl}_{3}\right)$;

${ }^{1} \mathrm{H}$ NMR (400 MHz, $\mathrm{CDCl}_{3}$ ) $\delta-0.03$ (s, 3H), - 0.02 (s, 3H), 0.84 (s, 3H), 2.29-2.41 (m, 2H), 3.52 (dd, $J=5.2$ and $10.8 \mathrm{~Hz}, 1 \mathrm{H}), 3.56(\mathrm{dd}, J=5.2$ and $10.8 \mathrm{~Hz}, 1 \mathrm{H}), 3.56(\mathrm{dd}, J=5.2$ and $10.8 \mathrm{~Hz}$, $1 \mathrm{H}), 4.08(\mathrm{dd}, J=5.2$ and $8.8 \mathrm{~Hz}, 1 \mathrm{H}), 4.59(\mathrm{dd}, J=7.2$ and $14.4 \mathrm{~Hz}, 1 \mathrm{H}), 4.66(\mathrm{dd}, J=8.8$ and $8.8 \mathrm{~Hz}, 1 \mathrm{H}), 4.89$ (ddt, $J=5.2,5.6$, and $6.8 \mathrm{~Hz}, 1 \mathrm{H}), 4.95(\mathrm{dd}, J=5.2$ and $8.8 \mathrm{~Hz}, 1 \mathrm{H}), 6.68(\mathrm{~d}, J$ $=14.4 \mathrm{~Hz}, 1 \mathrm{H}), 7.19-7.32(\mathrm{~m}, 5 \mathrm{H}), 7.41-7.45(\mathrm{~m}, 2 \mathrm{H}), 7.54-7.56(\mathrm{~m}, 1 \mathrm{H}), 7.61-7.65(\mathrm{~m}, 2 \mathrm{H}) ;{ }^{13} \mathrm{C}$ NMR $\left(100 \mathrm{MHz}, \mathrm{CDCl}_{3}\right) \delta$-5.3, -5.2, 18.4, 26.0, 31.6, 58.8, 63.6, 70.7, 74.6, 108.1, 125.3, 126.0, $128.5,129.0,129.5,129.8,130.6,133.1,138.2,155.8$; IR (thin film) $\mathrm{cm}^{-1} 2954 \mathrm{w}, 2929 \mathrm{w}, 2857 \mathrm{w}$, 1764s, 1719m, 1548s, 1493s, 1228s, 1042s; mass spectrum (APCI): m/e (\% relative intensity) $482(\mathrm{M}+\mathrm{H})^{+}(70), 360(56), 202(100), 150(20)$.

\section{General Procedure for the Bromine Mediated Pyran Formation.}

To a flame dried 5-mL RB-Flask was added enamide (200.0 $\mathrm{mg}, 0.53 \mathrm{mmol})$ and anhyd toluene $(5 \mathrm{~mL})$. Bromine $\left(0.5 \mathrm{M}\right.$ in $\left.\mathrm{CH}_{2} \mathrm{Cl}_{2}, 1.60 \mathrm{~mL}, 0.80 \mathrm{mmol}\right)$ was added dropwise over 15 min at $-78{ }^{\circ} \mathrm{C}$. The resulting reaction mixture was warmed up to room temperature and stirred for $30 \mathrm{~min}$, while being monitored via TLC. Upon disappearance of the starting material, the reaction mixture was concentrated in vacuo. Further purification was performed using silica gel flash column chromatography (gradient eluent: 4:1 to 1:1 hexane/EtOAc) to afford 3-bromo-2- 
pyran 7-Br with a diastereomeric ratio 7.4:1 and a combined yield of $83 \%$ (150.0 mg). Major diastereomer was separated via trituration with ethyl EtOAc:hexane as a white crystal $(90.0 \mathrm{mg}$, $50 \%)$.

\section{Characterizations.}

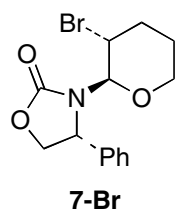

$R_{f}=0.38[33 \%$ EtOAc /hexane $] ; \mathrm{mp} 135-136^{\circ} \mathrm{C} ;[\alpha]_{\mathrm{D}}^{23}=-104.2\left(\mathrm{c} 0.58, \mathrm{CHCl}_{3}\right)$;

${ }^{1} \mathrm{H}$ NMR $\left(400 \mathrm{MHz}, \mathrm{CDCl}_{3}\right) \delta 1.47-1.54(\mathrm{~m}, 1 \mathrm{H}), 1.59$ (ddddd, $J=4.0,4.4,12.4,12.8$, and 12.8 $\mathrm{Hz}, 1 \mathrm{H}), 1.86$ (dddd, $J=4.8,11.6,12.8$, and $12.8 \mathrm{~Hz}, 1 \mathrm{H}), 2.41-2.47(\mathrm{~m}, 1 \mathrm{H}), 3.41$ (ddd, $J=2.8$, 11.6 , and $12.4 \mathrm{~Hz}, 1 \mathrm{H}$ ), 3.91(dddd, $J=1.6,2.0,4.4$, and $11.6 \mathrm{~Hz}, 1 \mathrm{H}$ ), 4.11 (dd, $J=8.4$ and 8.8 $\mathrm{Hz}, 1 \mathrm{H}), 4.48(\mathrm{~d}, J=10.0 \mathrm{~Hz}, 1 \mathrm{H}), 4.55-4.63(\mathrm{~m}, 1 \mathrm{H}), 4.61(\mathrm{dd}, J=8.8$ and $8.8 \mathrm{~Hz}, 1 \mathrm{H}), 5.06$ (dd, $J=8.4$ and $8.8 \mathrm{~Hz}, 1 \mathrm{H}), 7.28-7.35(\mathrm{~m}, 5 \mathrm{H}) ;{ }^{13} \mathrm{C} \mathrm{NMR}\left(100 \mathrm{MHz}, \mathrm{CDCl}_{3}\right) \delta$ 27.6, 35.7, 46.1, 59.4, 68.0, 70.6, 87.1, 128.0, 129.1, 129.3, 137.6, 157.6; IR (thin film) $\mathrm{cm}^{-1} 2954 \mathrm{w}, 2854 \mathrm{w}$, 1756s, 1413m, 1065s; mass spectrum (APCI): $\mathrm{m} / \mathrm{e}$ (\% relative intensity) $326[(\mathrm{M}+2)+\mathrm{H}]^{+}(54)$, $328(\mathrm{M}+\mathrm{H})^{+}(52), 246(100), 164$ (40).; HRMS (MALDI) calcd for $\mathrm{C}_{14} \mathrm{H}_{16} \mathrm{BrNNaO}_{3}[\mathrm{M}+\mathrm{Na}]^{+}$ 348.0206 , found 348.0222 .

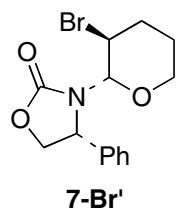

$R_{f}=0.27$ [33\% EtOAc/hexanes]; ${ }^{1} \mathrm{H}$ NMR $\left(500 \mathrm{MHz}, \mathrm{CDCl}_{3}\right) \delta 1.48-1.63(\mathrm{~m}, 2 \mathrm{H}), 1.82-1.91$ (m, 1H), $2.27(\mathrm{~m}, 1 \mathrm{H}), 3.41(\mathrm{td}, 1 \mathrm{H}, J=12.0,2.5 \mathrm{~Hz}), 3.48-3.53(\mathrm{~m}, 1 \mathrm{H}), 4.04(\mathrm{~m}, 1 \mathrm{H}), 4.19$ $(\mathrm{dd}, 1 \mathrm{H}, J=8.5,8.5 \mathrm{~Hz}), 4.62(\mathrm{dd}, 1 \mathrm{H}, J=9.0,9.0 \mathrm{~Hz}), 4.86(\mathrm{~d}, 1 \mathrm{H}, J=9.5 \mathrm{~Hz}), 5.09$ (dd, $1 \mathrm{H}, J$ $=8.5,8.5 \mathrm{~Hz}), 7.38-7.46(\mathrm{~m}, 3 \mathrm{H}), 7.50-7.52(\mathrm{~m}, 2 \mathrm{H}) ;{ }^{13} \mathrm{C} \mathrm{NMR}\left(125 \mathrm{MHz}, \mathrm{CDCl}_{3}\right) \delta 27.5$, 35.4, 47.9, 58.4, 68.4, 70.7, 87.1, 128.2, 129.1, 129.5, 138.0, and 158.3; IR (neat) $\mathrm{cm}^{-1} 3036 \mathrm{w}$, 2953w, 1759s, and 1393w; mass spectrum (APCI): m/e (\% relative intensity) $328(20)[(\mathrm{M}+2)+$ $\mathrm{H}]^{+}, 326(20)(\mathrm{M}+\mathrm{H})^{+}, 246(100)$; HRMS (MALDI) m/e calcd for $\mathrm{C}_{14} \mathrm{H}_{16} \mathrm{Br}^{79} \mathrm{NO}_{3} \mathrm{Na}^{+}(\mathrm{M}+$ $\left.\mathrm{Na}^{+}\right)$348.0206, found 348.0206 . 


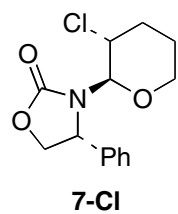

$R_{f}=0.51[33 \%$ EtOAc/hexane];

${ }^{1} \mathrm{H}$ NMR (400 MHz, $\left.\mathrm{CDCl}_{3}\right) \delta 1.57(\mathrm{~m}, 2 \mathrm{H}), 1.69(\mathrm{~m}, 1 \mathrm{H}), 2.35(\mathrm{~m}, 1 \mathrm{H}), 3.38(\mathrm{~m}, 1 \mathrm{H}), 3.88(\mathrm{ddt}$, $J=1.9,3.8$, and $11.2 \mathrm{~Hz}, 1 \mathrm{H}), 4.10(\mathrm{t}, J=8.5 \mathrm{~Hz}, 1 \mathrm{H}), 4.40(\mathrm{~d}, J=9.6 \mathrm{~Hz}, 1 \mathrm{H}), 4.50,(\mathrm{~m}, 1 \mathrm{H})$, $4.62(\mathrm{t}, J=8.7 \mathrm{~Hz}, 1 \mathrm{H}), 5.06,(\mathrm{t}, J=8.6 \mathrm{~Hz}, 1 \mathrm{H}), 7.42(\mathrm{~m}, 5 \mathrm{H}) ;{ }^{13} \mathrm{C} \mathrm{NMR}\left(100 \mathrm{MHz}, \mathrm{CDCl}_{3}\right) \delta$ 26.5, 34.8, 54.0, 59.5, 67.8, 70.8, 87.4, 127.9, 129.2, 129.3, 137.7, 157.6; IR (neat) $\mathrm{cm}^{-1} 2957 \mathrm{~m}$, $2858 \mathrm{~m}, 1761 \mathrm{~s}, 1416 \mathrm{~m}, 1071 \mathrm{~s}$; mass spectrum (APCI) for $\mathrm{C}_{14} \mathrm{H}_{16} \mathrm{ClNO}_{3}: \mathrm{m} / \mathrm{e}$ (\% relative intensity) $284[(\mathrm{M}+2)+\mathrm{H}]^{+}(28), 282(\mathrm{M}+\mathrm{H})^{+}(100), 246(55), 164$ (35).

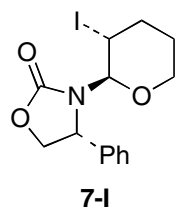

$R_{f}=0.28[20 \% \mathrm{EtOAc} / \mathrm{hexane}] ;[\alpha]_{\mathrm{D}}^{23}=-75.7\left(\mathrm{c} 0.65, \mathrm{CHCl}_{3}\right) ;$

${ }^{1} \mathrm{H}$ NMR $\left(400 \mathrm{MHz}, \mathrm{CDCl}_{3}\right) \delta 1.36(\mathrm{~d}, J=13.6 \mathrm{~Hz}, 1 \mathrm{H}), 1.48-1.63(\mathrm{~m}, 1 \mathrm{H}), 2.05(\mathrm{dq}, J=4.0$ and $12.8 \mathrm{~Hz}, 1 \mathrm{H}), 2.56(\mathrm{~d}, J=13.6 \mathrm{~Hz}, 1 \mathrm{H}), 3.46(\mathrm{dt}, J=2.0$ and $12.0 \mathrm{~Hz}, 1 \mathrm{H}), 4.00(\mathrm{dd}, J=4.4$ and $11.6 \mathrm{~Hz}, 1 \mathrm{H}), 4.14(\mathrm{t}, J=8.4 \mathrm{~Hz}, 1 \mathrm{H}), 4.53(\mathrm{~d}, J=9.2 \mathrm{~Hz}, 1 \mathrm{H}), 4.62(\mathrm{t}, J=8.4 \mathrm{~Hz}, 1 \mathrm{H}), 4.73$ (s, $1 \mathrm{H}), 5.03(\mathrm{t}, J=8.4 \mathrm{~Hz}, 1 \mathrm{H}), 7.27-7.56(\mathrm{~m}, 5 \mathrm{H}) ;{ }^{13} \mathrm{C} \mathrm{NMR}\left(100 \mathrm{MHz}, \mathrm{CDCl}_{3}\right) \delta 25.6,28.9$, $38.0,59.5,68.3,70.6,87.5,128.3,129.1,129.4,137.4,157.4$; IR (neat) $\mathrm{cm}^{-1} 2957 \mathrm{w}, 2859 \mathrm{w}$, 1766s, 1409m, 1360m, 1252m, 1213m; mass spectrum (APCI): m/e (\% relative intensity) 374 $(\mathrm{M}+\mathrm{H})^{+}(85), 246(100), 164(43)$

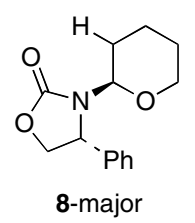

$R_{f}=0.24[20 \%$ EtOAc/hexane $] ;[\alpha]_{\mathrm{D}}^{23}=-129.5\left(\mathrm{c} 0.55, \mathrm{CHCl}_{3}\right)$;

${ }^{1} \mathrm{H}$ NMR $\left(400 \mathrm{MHz}, \mathrm{CDCl}_{3}\right.$ ) $\delta 0.98$ (ddddd, $J=4.0,4.4,11.6,12.0$, and $12.4 \mathrm{~Hz}, 1 \mathrm{H}$ ), 1.23-1.29 (m, $1 \mathrm{H}), 1.33-1.45(\mathrm{~m}, 2 \mathrm{H}), 1.63-1.70(\mathrm{~m}, 1 \mathrm{H}), 3.60(\mathrm{ddd}, J=3.6,11.2$, and $11.6 \mathrm{~Hz}, 1 \mathrm{H}), 4.06$ (dddd, $J=1.6,2.0,4.0$, and $11.6 \mathrm{~Hz}, 1 \mathrm{H}), 4.07(\mathrm{dd}, J=6.0$ and $8.8 \mathrm{~Hz}, 1 \mathrm{H}), 4.61(\mathrm{dd}, J=8.8$ and $9.2 \mathrm{~Hz}, 1 \mathrm{H}), 5.06(\mathrm{dd}, J=6.0$ and $9.2 \mathrm{~Hz}, 1 \mathrm{H}), 5.09(\mathrm{~d}, J=2.4 \mathrm{~Hz}, 1 \mathrm{H}), 7.33-7.40(\mathrm{~m}, 5 \mathrm{H}) ;{ }^{13} \mathrm{C}$ NMR $\left(100 \mathrm{MHz}, \mathrm{CDCl}_{3}\right)$ 23.6, 25.2, 31.0, 56.5, 68.6, 71.2, 83.6, 126.7, 128.8, 129.3, 141.4, 159.1; IR (neat) $\mathrm{cm}^{-1} 2947 \mathrm{w}, 2859 \mathrm{w}, 1756 \mathrm{~s}, 1406 \mathrm{~m}, 1220 \mathrm{~m}, 1080 \mathrm{~s}$; mass spectrum (APCI): m/e 
(\% relative intensity) $248(\mathrm{M}+\mathrm{H})^{+}(36), 164(100)$.

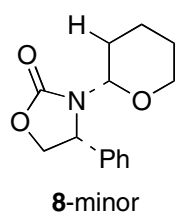

$R_{f}=0.22[20 \% \mathrm{EtOAc} /$ hexane $]$;

${ }^{1} \mathrm{H}$ NMR (400 MHz, $\mathrm{CDCl}_{3}$ ) $\delta$ 1.33-1.49 (m, 2H), 1.69 (ddddd, $J=2.0,2.0,2.4,2.4$, and $12.4 \mathrm{~Hz}$, $1 \mathrm{H}), 1.83-1.89(\mathrm{~m}, 1 \mathrm{H}), 2.12-2.22(\mathrm{~m}, 1 \mathrm{H}), 3.39$ (ddd, $J=4.4,10.8$ and $11.6 \mathrm{~Hz}, 1 \mathrm{H}), 3.89$ (dddd, $J=2.4,2.8,3.2$ and $11.6 \mathrm{~Hz}, 1 \mathrm{H}), 4.08(\mathrm{dd}, J=7.2$ and $8.8 \mathrm{~Hz}, 1 \mathrm{H}), 4.48(\mathrm{dd}, J=2.4$ and 11.2 $\mathrm{Hz}, 1 \mathrm{H}), 4.59(\mathrm{dd}, J=8.8$ and $8.8 \mathrm{~Hz}, 1 \mathrm{H}), 4.99(\mathrm{~d}, J=7.2$ and $8.8 \mathrm{~Hz}, 1 \mathrm{H}), 7.33-7.43(\mathrm{~m}, 5 \mathrm{H})$; mass spectrum (APCI): m/e (\% relative intensity) $248(\mathrm{M}+\mathrm{H})^{+}(30), 164(100)$.

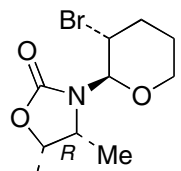

$\mathrm{Ph}$

$9-\mathrm{Br}$

$R_{f}=0.21[25 \% \mathrm{EtOAc} / \mathrm{hexane}] ;[\alpha]_{\mathrm{D}}^{23}=+3.10\left(\mathrm{c} 0.59, \mathrm{CHCl}_{3}\right)$;

${ }^{1} \mathrm{H}$ NMR $\left(400 \mathrm{MHz}, \mathrm{CDCl}_{3}\right) \delta 0.89(\mathrm{~d}, J=6.4 \mathrm{~Hz}, 3 \mathrm{H}), 1.63-1.71(\mathrm{~m}, 1 \mathrm{H}), 1.77$ (ddddd, $J=4.0$, 4.4, 12.4, 12.,8 and 12.8 Hz, 1H), 2.09 (dddd, $J=4.4,12.0,12.8$, and $12.8 \mathrm{~Hz}, 1 \mathrm{H}$ ), 2.53-2.61 (m, $1 \mathrm{H}$ ), 3.63 (ddd, $J=2.8,11.6$, and $12.4 \mathrm{~Hz}, 1 \mathrm{H}$ ), 4.05 (dddd, $J=2.4,2.8,4.4$, and $11.6 \mathrm{~Hz}, 1 \mathrm{H}$ ), $4.12(\mathrm{ddd}, J=2.0,10.4$, and $11.6 \mathrm{~Hz}, 1 \mathrm{H}), 4.25(\mathrm{dq}, J=6.4$ and $7.6 \mathrm{~Hz}, 1 \mathrm{H}), 5.10(\mathrm{~d}, J=10.4 \mathrm{~Hz}$, 1H), 7.30-7.41 (m, 5H); ${ }^{13} \mathrm{C}$ NMR $\left(100 \mathrm{MHz}, \mathrm{CDCl}_{3}\right) \delta 17.1$, ,27.9, 35.7, 46.9, 53.6, 68.3, 79.5, 86.6, 126.4, 126.6, 128.7, 134.7, 157.1; IR (neat) $\mathrm{cm}^{-1} 2974 \mathrm{w}, 2862 \mathrm{w}, 1761 \mathrm{~s}, 1457 \mathrm{~m}, 1069 \mathrm{~s}$; mass spectrum (APCI): m/e (\% relative intensity) $342[(\mathrm{M}+2)+\mathrm{H}]^{+}(18), 340(\mathrm{M}+\mathrm{H})^{+}(51)$, 260 (100), 216 (40), 178 (20).

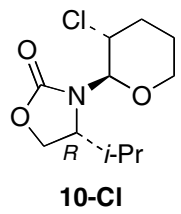

$R_{f}=0.46[33 \%$ EtOAc in hexane $] ;[\alpha]_{\mathrm{D}}^{23}=-28.3\left(\mathrm{c} 0.22, \mathrm{CHCl}_{3}\right)$;

${ }^{1} \mathrm{H} \mathrm{NMR}\left(400 \mathrm{MHz}, \mathrm{CDCl}_{3}\right) \delta 0.92(\mathrm{~d}, J=6.8 \mathrm{~Hz}, 3 \mathrm{H}), 1.00(\mathrm{~d}, J=6.8 \mathrm{~Hz}, 3 \mathrm{H}), 1.68(\mathrm{~m}, 1 \mathrm{H})$, $1.81(\mathrm{~m}, 2 \mathrm{H}), 2.04$ (d sept, $J=3.4$ and $6.8 \mathrm{~Hz}, 1 \mathrm{H}), 2.44(\mathrm{~m}, 1 \mathrm{H}), 3.53(\mathrm{td}, J=2.4$ and $11.7 \mathrm{~Hz}$, $1 \mathrm{H}), 3.88(\mathrm{dt}, J=3.7$ and $9.0 \mathrm{~Hz}, 1 \mathrm{H}), 4.06(\mathrm{~m}, 1 \mathrm{H}), 4.14(\mathrm{dd}, J=4.2$ and $9.0 \mathrm{~Hz}, 1 \mathrm{H}), 4.27(\mathrm{t}, J$ $=9.0 \mathrm{~Hz}, 1 \mathrm{H}), 4.47(\mathrm{~m}, 2 \mathrm{H}) ;{ }^{13} \mathrm{C} \mathrm{NMR}\left(100 \mathrm{MHz}, \mathrm{CDCl}_{3}\right) \delta 14.8,18.1,26.6,30.5,35.0,55.4$, 
60.9, 63.9, 68.4, 88.3, 157.8; IR (neat) $\mathrm{cm}^{-1} 2962 \mathrm{~m}, 2958 \mathrm{~m}, 1737 \mathrm{~s}, 1391 \mathrm{~m}, 1066 \mathrm{~s}$; mass spectrum (APCI): m/e (relative intensity) $250[(\mathrm{M}+2)+\mathrm{H}]^{+}(30), 248(\mathrm{M}+\mathrm{H})^{+}(100), 212(17)$, 130 (32).

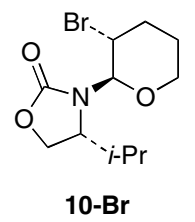

$R_{f}=0.19[25 \%$ EtOAc/hexane $] ; \operatorname{mp~} 127-128^{\circ} \mathrm{C} ;[\alpha]_{\mathrm{D}}^{23}=-43.1\left(\mathrm{c} 0.63, \mathrm{CHCl}_{3}\right)$;

${ }^{1} \mathrm{H}$ NMR $\left(400 \mathrm{MHz}, \mathrm{CDCl}_{3}\right) \delta 0.89(\mathrm{~d}, J=7.2 \mathrm{~Hz}, 3 \mathrm{H}), 0.94(\mathrm{~d}, J=7.2 \mathrm{~Hz}, 3 \mathrm{H}), 1.62-1.68$ (m, 1H), 1.72 (ddddd, $J=4.0,4.4,12.4,12.8$ and $12.8 \mathrm{~Hz}, 1 \mathrm{H}$ ), 2.07 (dddd, $J=4.8,12.4,12.8$ and $12.8 \mathrm{~Hz}, 1 \mathrm{H}$ ), 2.15 (dhept, $J=3.2$ and $7.2 \mathrm{~Hz}, 1 \mathrm{H}$ ), 2.51-2.58 (m, 1H), 3.61 (ddd, $J=3.2,12.0$, and $12.4 \mathrm{~Hz}, 1 \mathrm{H}), 3.89(\mathrm{ddd}, J=3.2,4.0$, and $8.8 \mathrm{~Hz}, 1 \mathrm{H}$ ), 4.03 (dddd, $J=1.6,2.0,4.4$ and 12.0 $\mathrm{Hz}, 1 \mathrm{H}), 4.10$ (ddd, $J=4.4,7.6$, and $10.0 \mathrm{~Hz}, 1 \mathrm{H}), 4.14(\mathrm{dd}, J=4.0$ and $8.8 \mathrm{~Hz}, 1 \mathrm{H}), 4.24(\mathrm{dd}, J$ $=8.8$ and $8.8 \mathrm{~Hz}, 1 \mathrm{H}), 4.97(\mathrm{~d}, J=10.0 \mathrm{~Hz}, 1 \mathrm{H}) ;{ }^{13} \mathrm{C} \mathrm{NMR}\left(100 \mathrm{MHz}, \mathrm{CDCl}_{3}\right) \delta$ 14.5, 18.2, 27.9, 30.3 , 35.7, 46.7, 58.0, 63.3, 68.3, 86.7, 158.0; IR (thin film) $\mathrm{cm}^{-1} 2964 \mathrm{w}, 2860 \mathrm{w}, 1742 \mathrm{~s}, 1423 \mathrm{~m}$, $1073 \mathrm{~m}$; mass spectrum (APCI): m/e (\% relative intensity) $294[(\mathrm{M}+2)+\mathrm{H}]^{+}(80), 292(\mathrm{M}+\mathrm{H})^{+}$ (88), 212 (100), 130 (70).

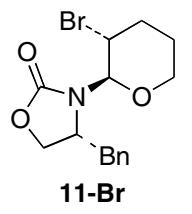

$R_{f}=0.20[25 \%$ EtOAc /hexane $\left.] ; \alpha\right]_{\mathrm{D}}^{23}=-65.4\left(\mathrm{c} 0.70, \mathrm{CHCl}_{3}\right) ;$

${ }^{1} \mathrm{H}$ NMR (400 MHz, $\mathrm{CDCl}_{3}$ ) $\delta 1.64-1.71(\mathrm{~m}, 1 \mathrm{H}), 1.74$ (ddddd, $J=4.0,4.4,12.4,12.8$ and 12.8 $\mathrm{Hz}, 1 \mathrm{H}$ ), 2.09 (dddd, $J=4.4,12.4,12.8$, and $12.8 \mathrm{~Hz}, 1 \mathrm{H}), 2.54-2.61$ (m, 1H), 2.83 (dd, $J=9.2$ and $13.6 \mathrm{~Hz}, 1 \mathrm{H}), 2.89(\mathrm{dd}, J=9.2$ and $13.6 \mathrm{~Hz}, 1 \mathrm{H}), 3.65(\mathrm{ddd}, J=2.8,11.6$, and $12.0 \mathrm{~Hz}, 1 \mathrm{H})$, 4.03-4.24 (m, 5H), $5.03(\mathrm{~d}, J=10.0 \mathrm{~Hz}, 1 \mathrm{H}), 7.20-7.35(\mathrm{~m}, 5 \mathrm{H}) ;{ }^{13} \mathrm{C} \mathrm{NMR}\left(100 \mathrm{MHz}, \mathrm{CDCl}_{3}\right) \delta$ 27.9, 36.6, 40.6, 46.8, 54.3, 67.1, 68.4, 86.8, 127.3, 129.1, 129.4, 136.1, 157.6; IR (neat) $\mathrm{cm}^{-1}$ 2957w, 2852w, 1754s, 1420m, 1068s; mass spectrum (APCI): m/e (\% relative intensity) 342 $[(\mathrm{M}+2)+\mathrm{H}]^{+}(60), 340(\mathrm{M}+\mathrm{H})^{+}(64), 260(100), 178(60)$.

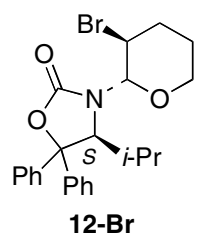


$R_{f}=0.42[33 \%$ EtOAc/hexanes $]$

$\mathrm{mp}=$ significant graying, then blackening prior to melting at $193-195{ }^{\circ} \mathrm{C}$;

$[\alpha]_{\mathrm{D}}^{25}=-90.0\left(c 1.4, \mathrm{CH}_{2} \mathrm{Cl}_{2}\right)$;

${ }^{1} \mathrm{H}$ NMR $\left(500 \mathrm{MHz}, \mathrm{CDCl}_{3}\right) \delta 0.71(\mathrm{~d}, J=6.5 \mathrm{~Hz}, 3 \mathrm{H}), 1.16(\mathrm{~d}, J=7.5 \mathrm{~Hz}, 3 \mathrm{H}), 1.59(\mathrm{~m}, 1 \mathrm{H})$, 1.68 (ddddd, $J=4.0,4.5,12.0,13.0$, and $14.0 \mathrm{~Hz}, 1 \mathrm{H}$ ), 1.95 (dddd, $J=4.5,11.5,13.0$, and13.0 $\mathrm{Hz}, 1 \mathrm{H}), 2.04$ (d sept, $J=1.0$ and $7.0 \mathrm{~Hz}, 1 \mathrm{H}), 2.47(\mathrm{~m}, 1 \mathrm{H}), 3.61$ (ddd, $J=2.5,12.0$, and 12.0 $\mathrm{Hz}, 1 \mathrm{H}$ ), 4.02 (dddd, $J=2.0,2.0,2.5$, and $11.5 \mathrm{~Hz}, 1 \mathrm{H}$ ), 4.10 (ddd, $J=4.5,10.0$, and $11.5 \mathrm{~Hz}$, $1 \mathrm{H}), 4.51(\mathrm{~d}, J=1.0 \mathrm{~Hz}, 1 \mathrm{H}), 4.93(\mathrm{~d}, J=10.0 \mathrm{~Hz}, 1 \mathrm{H}), 7.19-7.24(\mathrm{~m}, 2 \mathrm{H}), 7.30(\mathrm{~m}, 4 \mathrm{H}), 7.52$ $(\mathrm{m}, 2 \mathrm{H}), 7.64(\mathrm{~m}, 2 \mathrm{H})$; minor isomer $\delta 0.79(\mathrm{~d}, J=6.5 \mathrm{~Hz}, 3 \mathrm{H}), 1.09(\mathrm{~d}, J=7.5 \mathrm{~Hz}, 3 \mathrm{H}), 2.42(\mathrm{~m}$, $1 \mathrm{H}), 3.44$ (ddd, $J=12.0,12.0,2.5 \mathrm{~Hz}, 1 \mathrm{H}), 4.14(\mathrm{~m}, 1 \mathrm{H}), 4.46(\mathrm{~d}, J=1.0 \mathrm{~Hz}, 1 \mathrm{H}), 4.89(\mathrm{~m}, 1 \mathrm{H})$; ${ }^{13} \mathrm{C}$ NMR $\left(125 \mathrm{MHz}, \mathrm{CDCl}_{3}\right) \delta 14.8,21.5,27.6,30.0,36.1,45.3,67.9,68.5,86.7,88.7,125.6$, $126.4,127.7,128.1,128.3,128.5,139.2,144.4$, and 157.1; IR (thin film) $\mathrm{cm}^{-1} 3059 \mathrm{w}, 2944 \mathrm{w}$, 1749s, 1421m, 1258m, and 1075s; mass spectrum (APCI): m/e (\% relative intensity) 446 [(M+2) $+\mathrm{H}]^{+}(40), 444(\mathrm{M}+\mathrm{H})^{+}(40), 402(25), 400(25)$, and $364(100)$.

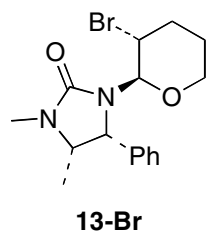

$R_{f}=0.47\left[50 \%\right.$ EtOAc /hexane]; mp 104-105 ${ }^{\circ} \mathrm{C} ;[\alpha]_{\mathrm{D}}{ }^{23}=-112.0\left(\mathrm{c} 0.70, \mathrm{CHCl}_{3}\right)$;

${ }^{1} \mathrm{H}$ NMR $\left(400 \mathrm{MHz}, \mathrm{CDCl}_{3}\right) \delta 0.73(\mathrm{~d}, J=6.4 \mathrm{~Hz}, 3 \mathrm{H}), 1.49$ (ddddd, $J=2.0,2.4,4.4$, 4.4, and $12.8 \mathrm{~Hz}, 1 \mathrm{H}$ ), 1.88 (ddddd, $J=4.0,4.4,12.4,12.8$, and $12.8 \mathrm{~Hz}, 1 \mathrm{H}$ ), 2.45 (dddd, $J=1.6,2.0$, 2.0, and $12.8 \mathrm{~Hz}, 1 \mathrm{H}), 2.79(\mathrm{~s}, 3 \mathrm{H}), 3.40(\mathrm{ddd}, J=2.0,12.0$, and $12.4 \mathrm{~Hz}, 1 \mathrm{H}), 3.78(\mathrm{dq}, J=6.4$ and $8.8 \mathrm{~Hz}, 1 \mathrm{H}), 3.90(\mathrm{ddd}, J=1.6,2.0,4.8$, and $12.0 \mathrm{~Hz}, 1 \mathrm{H}), 4.51(\mathrm{~d}, J=10.0 \mathrm{~Hz}, 1 \mathrm{H}), 4.76-$ $4.90(\mathrm{~m}, 1 \mathrm{H}), 4.93(\mathrm{~d}, J=8.8 \mathrm{~Hz}, 1 \mathrm{H}), 7.29-7.41(\mathrm{~m}, 5 \mathrm{H}) ;{ }^{13} \mathrm{C} \mathrm{NMR}\left(100 \mathrm{MHz}, \mathrm{CDCl}_{3}\right) \delta 14.7$, 27.9, 28.6, 36.2, 47.6, 55.9, 60.9, 67.8, 87.6, 128.2, 128.3, 128.9, 136.4, 160.3; IR (thin film) $\mathrm{cm}^{-1} 2973 \mathrm{w}, 2860 \mathrm{w}, 1707 \mathrm{~s}, 1435 \mathrm{~m}, 1060 \mathrm{~s}$; mass spectrum (APCI): m/e (\% relative intensity) 355 $[(\mathrm{M}+2)+\mathrm{H}]^{+}(62), 353(\mathrm{M}+\mathrm{H})^{+}(62), 273$ (100), 243 (22), 191 (18).

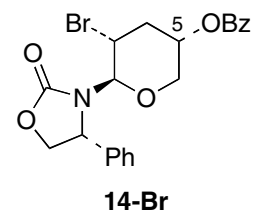

$R_{f}=0.44[33 \%$ EtOAc/hexane $]$;

$\mathrm{mp}=$ significant graying, then blackening prior to melting at $210-213{ }^{\circ} \mathrm{C}$; $[\alpha]_{\mathrm{D}}^{23}=-54.6\left(\mathrm{c} 0.13, \mathrm{CHCl}_{3}\right)$; 
${ }^{1} \mathrm{H}$ NMR (400 MHz, $\left.\mathrm{CDCl}_{3}\right) \delta 2.07(\mathrm{ddd}, J=11.2,12.4$, and $12.4 \mathrm{~Hz}, 1 \mathrm{H}), 2.90$ (dddd, $J=2.4$, 4.4, 4.4, and $12.4 \mathrm{~Hz}, 1 \mathrm{H}), 3.42(\mathrm{dd}, J=10.8$ and $10.8 \mathrm{~Hz}, 1 \mathrm{H}), 4.11-4.16(\mathrm{~m}, 1 \mathrm{H}), 4.15(\mathrm{dd}, J=$ 8.4 and $8.8 \mathrm{~Hz}, 1 \mathrm{H}), 4.54-4.72(\mathrm{~m}, 2 \mathrm{H}), 4.65(\mathrm{dd}, J=8.4$ and $8.8 \mathrm{~Hz}, 1 \mathrm{H}), 4.85$ (dddd, $J=4.4$, 5.2, 10.8, and $12.4 \mathrm{~Hz}, 1 \mathrm{H}), 5.08(\mathrm{dd}, J=8.4$ and $8.8 \mathrm{~Hz}, 1 \mathrm{H}), 7.39-7.47(\mathrm{~m}, 5 \mathrm{H}), 7.54-7.59(\mathrm{~m}$, 2H), $7.92(\mathrm{~m}, 3 \mathrm{H}) ;{ }^{13} \mathrm{C}$ NMR $\left(100 \mathrm{MHz}, \mathrm{CDCl}_{3}\right) \delta 40.4,41.3,59.3,67.0,68.7,70.8,86.8,127.9$, $128.7,129.3,129.5,129.6,129.8,133.6,137.3,127.4,165.4$; IR (thin film) $\mathrm{cm}^{-1} 2973 \mathrm{w}, 2907 \mathrm{w}$, 1760s, 1719s, 1452m, 1268s, 1069s; mass spectrum (APCI): m/e (\% relative intensity) 448 $[(\mathrm{M}+2)+\mathrm{H}]^{+}(16), 446(\mathrm{M}+\mathrm{H})^{+}(18), 285(70), 283(62), 105(100)$.

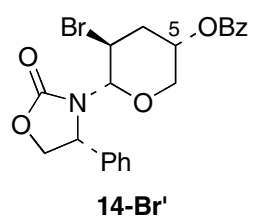

$R_{f}=0.40[33 \%$ EtOAc/hexane $]$

$\mathrm{mp}=$ significant graying, then blackening prior to melting at $208-210{ }^{\circ} \mathrm{C}$;

$[\alpha]_{\mathrm{D}}^{23}=-162.7\left(\mathrm{c} 0.44, \mathrm{CHCl}_{3}\right)$;

${ }^{1} \mathrm{H}$ NMR (400 MHz, $\mathrm{CDCl}_{3}$ ) $\delta 2.21(\mathrm{ddd}, J=2.8,12.4$, and $14.4 \mathrm{~Hz}, 1 \mathrm{H}), 2.56$ (dddd, $J=2.4$, $2.8,4.8$, and $14.4 \mathrm{~Hz}, 1 \mathrm{H}), 3.62-3.76(\mathrm{~m}, 1 \mathrm{H}), 3.84(\mathrm{dd}, J=0.8$ and $12.8 \mathrm{~Hz}, 1 \mathrm{H}), 4.22(\mathrm{dd}, J=$ 8.8 and $8.8 \mathrm{~Hz}, 1 \mathrm{H}), 4.25(\mathrm{ddd}, J=2.0,2.8$, and $12.8 \mathrm{~Hz}, 1 \mathrm{H}), 4.64(\mathrm{dd}, J=8.8$ and $8.8 \mathrm{~Hz}, 1 \mathrm{H})$, 5.00 (dddd, $J=0.8,2.0,2.8$, and $12.8 \mathrm{~Hz}, 1 \mathrm{H}), 5.12(\mathrm{~d}, J=10.8 \mathrm{~Hz} 1 \mathrm{H}), 5.16$ (dd, $J=8.8$ and $8.8 \mathrm{~Hz}, 1 \mathrm{H}), 7.36-7.42(\mathrm{~m}, 3 \mathrm{H}), 7.48-7.52(\mathrm{~m}, 2 \mathrm{H}), 7.54-7.56(\mathrm{~m}, 2 \mathrm{H}), 7.62-7.66(\mathrm{~m}, 1 \mathrm{H}), 7.92-$ $7.94(\mathrm{~m}, 2 \mathrm{H}) ;{ }^{13} \mathrm{C}$ NMR $\left(100 \mathrm{MHz}, \mathrm{CDCl}_{3}\right) \delta$ 39.1, 43.7, 57.8, 69.2, 69.9, 70.8, 86.8, 128.4, 128.6, 129.2, 129.7, 129.9, 130.0, 133.7, 137.8, 158.7, 165.6; IR (thin film) $\mathrm{cm}^{-1} 3109 \mathrm{w}, 2994 \mathrm{w}$, 1759s, 1720s, 1420m, 1273s, 1028s, 1016s; mass spectrum (APCI): m/e (\% relative intensity) $448[(\mathrm{M}+2)+\mathrm{H}]^{+}(60), 446(\mathrm{M}+\mathrm{H})^{+}(52), 285$ (52), 283 (60), 164 (100), 105 (74).

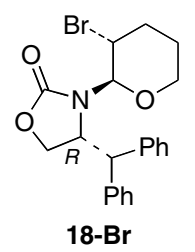

$R_{f}=0.20[25 \%$ EtOAc/hexane $] ;[\alpha]_{\mathrm{D}}^{23}=-125.3\left(\mathrm{c} 0.63, \mathrm{CHCl}_{3}\right)$;

${ }^{1} \mathrm{H}$ NMR (500 MHz, $\left.\mathrm{CDCl}_{3}\right) \delta 1.50-1.54$ (m, 1H), 1.55 (ddddd, $J=4.0,4.5,12.0,12.0$ and 12.5 $\mathrm{Hz}, 1 \mathrm{H}$ ), 1.82 (dddd, $J=7.0,11.5,11.5$, and $12.0 \mathrm{~Hz}, 1 \mathrm{H}$ ), 2.41 (dddd, $J=2.0,2.5,4.5$, and 11.5 $\mathrm{Hz}, 1 \mathrm{H}$ ), 3.88 (dddd, $J=2.5,12.0,12.0$, and $12.5 \mathrm{~Hz}, 1 \mathrm{H}$ ), 4.02 (dddd, $J=2.0,2.5,4.0$, and 12.0 $\mathrm{Hz}, 1 \mathrm{H}), 4.13(\mathrm{dd}, J=5.0$ and $9.0 \mathrm{~Hz}, 1 \mathrm{H}), 4.17-4.31(\mathrm{~m}, 1 \mathrm{H}), 4.34(\mathrm{dd}, J=8.5$ and $9.0 \mathrm{~Hz}, 1 \mathrm{H})$, $4.51(\mathrm{~d}, J=5.5 \mathrm{~Hz}, 1 \mathrm{H}), 4.64-4.74(\mathrm{~m}, 1 \mathrm{H}), 4.79$ (ddd, $J=5.0,5.5$, and $8.5 \mathrm{~Hz}, 1 \mathrm{H}), 7.19-7.35$ 
(m, 8H), 7.36-7.39 (m, 2H); ${ }^{13} \mathrm{C}$ NMR (100 MHz, $\left.\mathrm{CDCl}_{3}\right) \delta 27.6,35.6,46.9,54.1,56.5,65.9$, $68.2,87.1,127.4,127.5,128.6,128.9,128.9,129.1,139.8,141.4,157.7$; IR (neat) $\mathrm{cm}^{-1} 2957 \mathrm{w}$, 2857w, 1760s, 1424m, 1067s; mass spectrum (APCI): m/e (\% relative intensity) $418[(\mathrm{M}+2)+$ $\mathrm{H}]^{+}(60), 416(\mathrm{M}+\mathrm{H})^{+}(64), 336(40), 254(70)$.

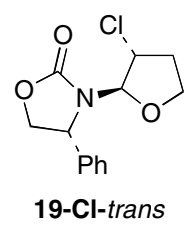

$R_{f}=0.40[50 \% \mathrm{EtOAc} /$ hexane $] ; \operatorname{mp} 98-100{ }^{\circ} \mathrm{C} ;[\alpha]_{\mathrm{D}}^{23}=-197.0\left(\mathrm{c} 0.21, \mathrm{CH}_{2} \mathrm{Cl}_{2}\right)$;

${ }^{1} \mathrm{H}$ NMR $\left(400 \mathrm{MHz}, \mathrm{CDCl}_{3}\right.$ ) $\delta 2.11$ (dddd, $J=5.2,6.0,15.2$, and $18.4 \mathrm{~Hz}, 1 \mathrm{H}$ ), 2.69 (dddd, $J$ $=6.8,6.8,14.0$, and $20.0 \mathrm{~Hz}, 1 \mathrm{H},), 3.94-4.06(\mathrm{~m}, 2 \mathrm{H}), 4.10(\mathrm{t}, J=8.8 \mathrm{~Hz}, 1 \mathrm{H}), 4.62(\mathrm{t}, J=8.8 \mathrm{~Hz}$, $1 \mathrm{H}), 4.83(\mathrm{~d}, J=4.0 \mathrm{~Hz}, 1 \mathrm{H}), 4.94(\mathrm{t}, J=8.8 \mathrm{~Hz}, 1 \mathrm{H}), 5.12(\mathrm{ddd}, J=4.4,4.8$, and $8.8 \mathrm{~Hz}, 1 \mathrm{H})$, 7.38-7.457 (m, 5H); ${ }^{13} \mathrm{C}$ NMR (125 MHz, $\left.\mathrm{CDCl}_{3}\right) \delta 36.3,57.3,61.1,68.0,70.5,92.6,127.6$, 129.60, 129.62, 137.2, 157.3; IR (thin film) cm-1 3037m, 2990m, 1763s, 1416m, 1340m, 1263s; mass spectrum (APCI): m/e (\% relative intensity) $270[(\mathrm{M}+2)+\mathrm{H}]^{+}(13), 268(\mathrm{M}+\mathrm{H})^{+}(42)$, 164 (20), 102 (100).

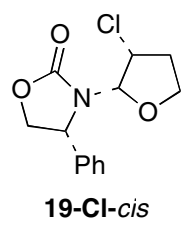

$R_{f}=0.32[50 \% \mathrm{EtOAc} /$ hexane $]$

${ }^{1} \mathrm{H}$ NMR (400 MHz, $\mathrm{CDCl}_{3}$ ) $\delta 1.84$ (dddd, $J=8.8,8.8,12.4$, and $17.2 \mathrm{~Hz}, 1 \mathrm{H}$ ), 2.20 (dddd, $J$ =3.6, 7.2, 10.4, and $16.0 \mathrm{~Hz}, 1 \mathrm{H}$, ), 3.71(ddd, $J=6.8,8.4$, and $15.2 \mathrm{~Hz}, 1 \mathrm{H}$ ), 4.05 (ddd, $J=3.6$, 8.4, and $11.6 \mathrm{~Hz}, 1 \mathrm{H}), 4.19(\mathrm{dd}, J=7.2$ and $8.4 \mathrm{~Hz}, 1 \mathrm{H}), 4.37$ (ddd, $J=6.0,7.6$, and $13.6 \mathrm{~Hz}$, $1 \mathrm{H}), 4.68(\mathrm{t}, J=8.8 \mathrm{~Hz}, 1 \mathrm{H}), 5.00(\mathrm{dd}, J=7.6$ and $8.8 \mathrm{~Hz}, 1 \mathrm{H}), 5.30(\mathrm{~d}, J=6.0 \mathrm{~Hz}, 1 \mathrm{H}), 7.32-$ 7.50 (m, 5H); IR (neat) $\mathrm{cm}^{-1} 3030 \mathrm{w}, 2908 \mathrm{~m}, 2367 \mathrm{~m}, 1750 \mathrm{~s}, 1572 \mathrm{~m}, 1476 \mathrm{~m}, 1334 \mathrm{~m}$; mass spectrum (APCI): m/e (\% relative intensity) $270[(\mathrm{M}+2)+\mathrm{H}]^{+}(13), 268(\mathrm{M}+\mathrm{H})^{+}(42), 164(20)$, 102 (100).

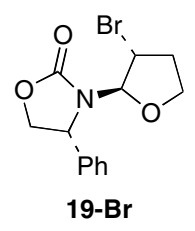

$R_{f}=0.12[33 \%$ EtOAc/hexane $] ; \mathrm{mp} 99-101{ }^{\circ} \mathrm{C} ;[\alpha]_{\mathrm{D}}^{23}=-87.0\left(\mathrm{c} 0.78, \mathrm{CH}_{2} \mathrm{Cl}_{2}\right)$; 
${ }^{1} \mathrm{H}$ NMR $\left(500 \mathrm{MHz}, \mathrm{CDCl}_{3}\right.$ ) $\delta 2.08$ (dddd, $J=6.5,6.5,13.0$, and $19.0 \mathrm{~Hz}, 1 \mathrm{H}$ ), 2.73 (dddd, $J=$ 7.0, 7.0, 14.0, and $21.0 \mathrm{~Hz}, 1 \mathrm{H}), 3.92-4.02(\mathrm{~m}, 2 \mathrm{H}), 4.10(\mathrm{t}, J=8.5 \mathrm{~Hz}, 1 \mathrm{H}), 4.62(\mathrm{t}, J=8.5 \mathrm{~Hz}$, $1 \mathrm{H}), 4.96(\mathrm{t}, J=8.5 \mathrm{~Hz}, 1 \mathrm{H}), 4.99(\mathrm{~d}, J=4.0 \mathrm{~Hz}, 1 \mathrm{H}), 5.12$ (ddd, $J=4.5,5.5$, and $10.0 \mathrm{~Hz}, 1 \mathrm{H}$ ), 7.38-7.45 (m, 5H); ${ }^{13} \mathrm{C}$ NMR (100 MHz, $\left.\mathrm{CDCl}_{3}\right) \delta 36.8,46.0,61.0,68.1,70.5,92.8,127.5,129.5$, 129.6, 137.2, 157.3; IR (neat) $\mathrm{cm}^{-1} 3057 \mathrm{~m}, 2983 \mathrm{~m}, 2900 \mathrm{~m}, 2360 \mathrm{~m}, 1731 \mathrm{~s}, 1415 \mathrm{~s}, 1288 \mathrm{~s}$; mass spectrum (APCI): m/e (\% relative intensity) $314\left[(\mathrm{M}+2)+\mathrm{H}^{+}(29), 312(\mathrm{M}+\mathrm{H})^{+}(30), 164\right.$ (100).

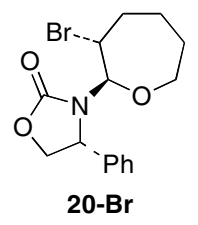

$R_{f}=0.40[25 \%$ EtOAc/hexane $] ;[\alpha]_{\mathrm{D}}^{23}=-68.8\left(\mathrm{c} 2.61, \mathrm{CCl}_{4}\right)$;

${ }^{1} \mathrm{H}$ NMR $\left(500 \mathrm{MHz}, \mathrm{CDCl}_{3}\right) \delta$ 1.56-1.59 (m, 2H), 1.67-1.72 (m, 2H), 1.99-2.06 (m, 1H), 2.27$2.34(\mathrm{~m}, 1 \mathrm{H}), 3.52(\mathrm{ddd}, J=5.5,5.5$, and $11.5 \mathrm{~Hz}, 1 \mathrm{H}), 4.00(\mathrm{ddd}, J=5.5,5.5$, and $11.5 \mathrm{~Hz}, 1 \mathrm{H}$ ), $3.52(\mathrm{dd}, J=8.5$ and $8.5 \mathrm{~Hz}, 1 \mathrm{H}), 4.10(\mathrm{dd}, J=8.5$ and $8.5 \mathrm{~Hz}, 1 \mathrm{H}), 4.61(\mathrm{dd}, J=8.5 \mathrm{and} 8.5 \mathrm{~Hz}$, $1 \mathrm{H}), 4.62(\mathrm{~d}, J=9.0 \mathrm{~Hz}, 1 \mathrm{H}), 5.08(\mathrm{ddd}, J=4.5,7.5 \mathrm{and} 9.0 \mathrm{~Hz}, 1 \mathrm{H}), 7.25-7.50(\mathrm{~m}, 5 \mathrm{H}) ;{ }^{13} \mathrm{C}$ NMR $\left(125 \mathrm{MHz}, \mathrm{CDCl}_{3}\right) \delta 23.5,30.2,36.7,50.6,60.0,70.6,70.6,91.0,128.2,128.2,129.3$, 129.3, 129.4, 137.2, 157.9; IR (neat) $\mathrm{cm}^{-1} 2981 \mathrm{w}, 2860 \mathrm{brs}, 2342 \mathrm{brs}, 1750 \mathrm{~s}, 1216 \mathrm{~s}, 1159 \mathrm{~s}, 1084 \mathrm{~s}$, 1043s; mass spectrum $(\mathrm{APCI})_{3}: \mathrm{m} / \mathrm{e}$ (\%relative intensity) $342[(\mathrm{M}+2)+\mathrm{H}]^{+}(18), 340(\mathrm{M}+\mathrm{H})^{+}$ (18), $260.1(100)$.

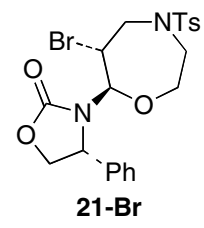

$R_{f}=0.29[50 \%$ EtOAc/hexanes $] ;[\alpha]_{\mathrm{D}}^{23}=-40.1\left(\mathrm{c} 1.77, \mathrm{CH}_{2} \mathrm{Cl}_{2}\right)$;

${ }^{1} \mathrm{H}$ NMR $\left(500 \mathrm{MHz}, \mathrm{CDCl}_{3}\right) \delta 2.42(\mathrm{~s}, 3 \mathrm{H}), 3.01(\mathrm{ddd}, J=4.0,10.0$, and $13.5 \mathrm{~Hz}, 1 \mathrm{H}), 3.40$ (ddd, $J=3.0,3.0$, and $13.5 \mathrm{~Hz}, 1 \mathrm{H}), 3.54(\mathrm{ddd}, J=3.5,10.0$, and $13.0 \mathrm{~Hz}, 1 \mathrm{H}), 3.63(\mathrm{dd}, J=3.5$ and $15.5 \mathrm{~Hz}, 1 \mathrm{H}), 3.79(\mathrm{dd}, \mathrm{J}=5.0$ and $15.5 \mathrm{~Hz}, 1 \mathrm{H}), 4.01(\mathrm{ddd}, J=3.5,3.5$, and $13.0 \mathrm{~Hz}, 1 \mathrm{H}), 4.12$ (dd, $J=9.0$ and $9.0 \mathrm{~Hz}, 1 \mathrm{H}), 4.60(\mathrm{dd}, J=8.5$ and $8.5 \mathrm{~Hz}, 1 \mathrm{H}), 4.64(\mathrm{~d}, J=9.0 \mathrm{~Hz}, 1 \mathrm{H}), 4.95(\mathrm{dd}$, $J=8.5$ and $8.5 \mathrm{~Hz}, 1 \mathrm{H}), 5.12(\mathrm{ddd}, J=4.0,4.0$, and $9.0 \mathrm{~Hz}, 1 \mathrm{H}), 7.28(\mathrm{~d}, J=8.0 \mathrm{~Hz}, 2 \mathrm{H}), 7.39-$ $7.44(\mathrm{~m}, 5 \mathrm{H})$, and $7.63(\mathrm{~d}, J=8.5 \mathrm{~Hz}, 2 \mathrm{H})$; minor isomer: $\delta 2.44(\mathrm{~s}, 3 \mathrm{H}), 3.29(\mathrm{ddd}, J=13.5$, 11.0, $3.5 \mathrm{~Hz}, 1 \mathrm{H}), 3.45(\mathrm{dd}, J=15.5,4.5 \mathrm{~Hz}, 1 \mathrm{H}), 3.92(\mathrm{ddd}, J=13.0,4.5,2.5 \mathrm{~Hz}, 1 \mathrm{H}), 4.06(\mathrm{dd}$, $J=6.5,6.5 \mathrm{~Hz}, 1 \mathrm{H}), 4.22(\mathrm{dd}, J=9.0,7.5 \mathrm{~Hz}, 1 \mathrm{H}), 4.38(\mathrm{~m}, 1 \mathrm{H}), 4.66(\mathrm{dd}, J=9.0,9.0 \mathrm{~Hz}, 1 \mathrm{H})$, $4.89(\mathrm{~d}, J=9.5 \mathrm{~Hz}, 1 \mathrm{H})$, and $4.99(\mathrm{dd}, J=9.0,7.5 \mathrm{~Hz}, 1 \mathrm{H}) ;{ }^{13} \mathrm{C} \mathrm{NMR}\left(125 \mathrm{MHz}, \mathrm{CDCl}_{3}\right) \delta 21.7$, 
46.8, 51.5, 53.9, 60.1, 69.9, 70.5, 90.8, 127.5, 128.0, 129.4, 129.7, 130.1, 135.3, 136.3, 144.1, and 157.6; IR (neat) $\mathrm{cm}^{-1} 3064 \mathrm{w}, 2919 \mathrm{w}, 1755 \mathrm{~s}, 1339 \mathrm{~m}, 1160 \mathrm{~s}, 1082 \mathrm{~m}, 1026 \mathrm{~s}$, and 906m; mass spectrum (APCI): m/e (\% relative intensity) $497[(\mathrm{M}+2)+\mathrm{H}]^{+}(20), 495(\mathrm{M}+\mathrm{H})^{+}(20), 415$ (100); HRMS (MALDI) m/e calcd for $\mathrm{C}_{21} \mathrm{H}_{23} \mathrm{BrN}_{2} \mathrm{O}_{5} \mathrm{SNa}+[\mathrm{M}+\mathrm{Na}]^{+}$517.0403, found 517.0541.

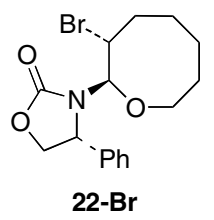

$R_{f}=0.23[30 \% \mathrm{EtOAc} /$ hexane $] ;[\alpha]_{\mathrm{D}}^{23}=-46.0\left(c 1.53, \mathrm{CHCl}_{3}\right)$;

${ }^{1} \mathrm{H}$ NMR (400 MHz, $\left.\mathrm{CDCl}_{3}\right) \delta$ 1.02-1.38 (m, 6H), 1.72-1.80 (m, 1H), 1.87-1.96 (m, 1H), 3.31$3.41(\mathrm{~m}, 2 \mathrm{H}), 4.18-4.27(\mathrm{~m}, 2 \mathrm{H}), 4.66(\mathrm{t}, J=8.8 \mathrm{~Hz}, 1 \mathrm{H}), 4.90(\mathrm{dd}, J=6.0$ and $9.2 \mathrm{~Hz}, 1 \mathrm{H}), 5.14$ $(\mathrm{d}, J=10.0 \mathrm{~Hz}, 1 \mathrm{H}) 7.31-7.39(\mathrm{~m}, 5 \mathrm{H}) ;{ }^{13} \mathrm{C} \mathrm{NMR}\left(100 \mathrm{MHz}, \mathrm{CDCl}_{3}\right) \delta 22.8,25.3,28.3,34.6$, 52.0, 56.8, 66.6, 70.9, 85.2, 127.2, 129.0, 129.1, 139.9, 158.8; IR (neat) $\mathrm{cm}^{-1} 2940 \mathrm{w}, 1750 \mathrm{~s}$, $1478 \mathrm{w}, 1458 \mathrm{~m}, 1224 \mathrm{~m}, 1056 \mathrm{~m}$; mass spectrum (APCI): $\mathrm{m} / \mathrm{e}$ (\% relative intensity) $356[(\mathrm{M}+2)+$ $\mathrm{H}]^{+}(100), 354(\mathrm{M}+\mathrm{H})^{+}(100), 274(30)$.

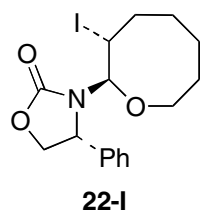

$R_{f}=0.25[30 \% \mathrm{EtOAc} /$ hexane $] ;[\alpha]_{\mathrm{D}}^{23}=-48.9\left(c 0.43, \mathrm{CHCl}_{3}\right)$;

${ }^{1} \mathrm{H}$ NMR $\left(400 \mathrm{MHz}, \mathrm{CDCl}_{3}\right) \delta$ 0.99-1.13 (m, 2H), 1.16-1.40 (m, 4H), 1.68-1.85 (m, 2H), 3.27 (m, $1 \mathrm{H}), 3.36(\mathrm{~m}, 1 \mathrm{H}), 4.25(\mathrm{dd}, J=4.8$ and $8.8 \mathrm{~Hz}, 1 \mathrm{H}), 4.34(\mathrm{dt}, J=3.6$ and $10.4 \mathrm{~Hz}, 1 \mathrm{H}), 4.67(\mathrm{t}$, $J=8.8 \mathrm{~Hz}, 1 \mathrm{H}), 4.85(\mathrm{dd}, J=5.2$ and $9.2 \mathrm{~Hz}, 1 \mathrm{H}), 5.17(\mathrm{~d}, J=10.4 \mathrm{~Hz}, 1 \mathrm{H}), 7.31-7.38(\mathrm{~m}, 5 \mathrm{H})$; ${ }^{13} \mathrm{C} \mathrm{NMR}\left(100 \mathrm{MHz}, \mathrm{CDCl}_{3}\right) \delta$ 25.0, 25.2, 28.5, 33.2, 35.9, 56.6, 66.5, 70.9, 85.5, 127.2, 128.9, 129.1, 140.1, 158.6; IR (neat) $\mathrm{cm}^{-1} 2927 \mathrm{w}, 2360 \mathrm{w}, 1749 \mathrm{~s}, 1476 \mathrm{w}, 1401 \mathrm{~m}, 1221 \mathrm{~m}, 1041 \mathrm{~m}$; mass spectrum (APCI): m/e (\% relative intensity) $402(\mathrm{M}+\mathrm{H})^{+}(100), 274(50)$.

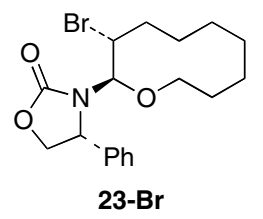

$R_{f}=0.33[25 \% \mathrm{EtOAc} / \mathrm{hexane}] ;[\alpha]_{\mathrm{D}}^{23}=-59.1\left(\mathrm{c} 0.24, \mathrm{CCl}_{4}\right)$;

${ }^{1} \mathrm{H}$ NMR (400 MHz, $\left.\mathrm{CDCl}_{3}\right) \delta 1.00-1.44(\mathrm{~m}, 10 \mathrm{H}), 1.76(\mathrm{~m} 1 \mathrm{H}), 1.86(\mathrm{~m}, 1 \mathrm{H}), 3.28(\mathrm{~m}, 2 \mathrm{H})$, $4.17(\mathrm{ddd}, \mathrm{J}=3.2,4.8$, and $10.0 \mathrm{~Hz}, 1 \mathrm{H}), 4.23(\mathrm{dd}, \mathrm{J}=4.8$ and $8.8 \mathrm{~Hz}, 1 \mathrm{H}), 4.66(\mathrm{dd}, \mathrm{J}=8.8$ and 
$8.8 \mathrm{~Hz}), 4.91(\mathrm{dd}, \mathrm{J}=5.2$ and $8.8 \mathrm{~Hz}, 1 \mathrm{H}), 5.13(\mathrm{~d}, \mathrm{~J}=10.0 \mathrm{~Hz}, 1 \mathrm{H}), 7.15-7.55(\mathrm{~m}, 5 \mathrm{H}) ;{ }^{13} \mathrm{C}$ NMR $\left(100 \mathrm{MHz} \mathrm{CDCl}_{3}\right) \delta$ 25.1, 26.3, 28.7, 29.0, 30.1, 34.6, 52.7, 56.9, 68.0, 70.9, 85.9, 127.2, 127.2, 129.0, 129.1, 129.1, 140.0, 158.8; IR (neat) $\mathrm{cm}^{-1} 3018 \mathrm{w}, 2981 \mathrm{w}, 2932 \mathrm{w}, 2859 \mathrm{w}, 2360 \mathrm{~s}$, 2341s, 1792s, 1215s, 1084br; mass spectrum (APCI): m/e (\% relative intensity) $384[(\mathrm{M}+2)+$ $\mathrm{H}]^{+}(97), 382(\mathrm{M}+\mathrm{H})^{+}(100), 302(14), 260(9)$;

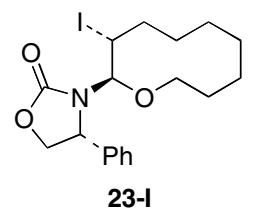

$R_{f}=0.33[25 \%$ EtOAc/hexane $] ;[\alpha]_{\mathrm{D}}^{23}=-59.6\left(\mathrm{c} 0.82, \mathrm{CCl}_{4}\right)$;

${ }^{1} \mathrm{H}$ NMR (400 MHz, $\left.\mathrm{CDCl}_{3}\right) \delta$ 1.01-1.42 (m, 10H), $1.82(\mathrm{~m}, 1 \mathrm{H}), 1.91(\mathrm{~m}, 1 \mathrm{H}), 3.28(\mathrm{~m}, 2 \mathrm{H})$, $4.26(\mathrm{dd}, J=4.4$ and $8.8 \mathrm{~Hz}, 1 \mathrm{H}), 4.30(\mathrm{~m}, 1 \mathrm{H}), 4.69(\mathrm{dd}, J=8.8$ and $8.8 \mathrm{~Hz}, 1 \mathrm{H}), 4.86(\mathrm{dd}, J=$ 4.8 and $8.8 \mathrm{~Hz}, 1 \mathrm{H}), 5.18(\mathrm{~d}, J=11.2 \mathrm{~Hz}, 1 \mathrm{H}), 7.15-7.60(\mathrm{~m}, 5 \mathrm{H}) ;{ }^{13} \mathrm{C} \mathrm{NMR}\left(100 \mathrm{MHz}, \mathrm{CDCl}_{3}\right)$ $\delta$ 26.0, 27.2, 28.7, 29.1, 30.0, 33.9, 35.8, 56.6, 67.9, 70.9, 86.2, 127.2, 127.2, 129.1, 129.2, 129.2, 140.1, 158.6; IR (neat) $\mathrm{cm}^{-1} 3016 \mathrm{w}, 2930 \mathrm{w}, 2857 \mathrm{w}, 2360 \mathrm{~s}, 2341 \mathrm{~s}, 1749 \mathrm{~s}, 1216 \mathrm{~s}, 1082 \mathrm{~s}$; mass spectrum (APCI): m/e (\% relative intensity) $430(\mathrm{M}+\mathrm{H})^{+}(100), 338(38), 302(8)$. 